\title{
Large impact features on middle-sized icy satellites
}

\author{
Jeffrey M. Moore ${ }^{\mathrm{a}, *}$, Paul M. Schenk ${ }^{\mathrm{b}}$, Lindsey S. Bruesch ${ }^{\mathrm{c}}$, Erik Asphaug ${ }^{\mathrm{c}}$, \\ William B. McKinnon ${ }^{\mathrm{d}}$ \\ a Space Sciences Division, NASA Ames Research Center, MS 245-3, Moffett Field, CA 94035-1000, USA \\ ${ }^{\mathrm{b}}$ Lunar and Planetary Institute, 3600 Bay Area Blvd., Houston, TX 77058, USA \\ c Earth Sciences Department, University of California, Santa Cruz, CA 95064, USA \\ d Department of Earth and Planetary Sciences and McDonnell Center for the Space Sciences, Washington University, Saint Louis, MO 63130, USA
}

Received 7 November 2003; revised 12 May 2004

Available online 2 July 2004

\begin{abstract}
All of the large impact features of the middle-sized icy satellites of Saturn and Uranus that were clearly observed by the Voyager spacecraft are described. New image mosaics and stereo-and-photoclinometrically-derived digital elevation models are presented. Landforms related to large impact features, such as secondary craters and possible antipodal effects are examined and evaluated. Of the large impacts, Odysseus on Tethys appears to have had the most profound effect on its "target" satellite of any of the impact features we examined. Our modeling suggests that the Odysseus impact may have caused the prompt formation of Ithaca Chasma, a belt of tectonic troughs that roughly follow a great circle normal to the center of Odysseus, although other hypotheses remain viable. We identify probable secondary cratering from Tirawa on Rhea. We attribute a number of converging coalescing crater chains on Rhea to a putative, possibly relatively fresh, $\sim 350 \mathrm{~km}$-diameter impact feature. We examine the antipodes of Odysseus, the putative $\sim 350 \mathrm{~km}$-diameter Rhean impact feature, and Tirawa, and conclude that evidence from Voyager data for damage from seismic focusing is equivocal, although our modeling results indicate that such damage may have occurred. We propose a number of observations and tests for Cassini that offer the opportunity to differentiate among the various explanations and speculations reviewed and evaluated in this study.
\end{abstract}

Published by Elsevier Inc.

Keywords: Satellites of Saturn; Satellites of Uranus; Impact processes; Surfaces, satellite

\section{Introduction}

Orbiting Saturn and Uranus are a number of satellites which, because of their size and composition, form a distinct group of objects in the Solar System referred to in this study as middle-sized, icy satellites (McKinnon, 1999). All of the satellites in this group have densities of less than $1700 \mathrm{~kg} \mathrm{~m}^{-3}$, implying that they are mostly composed of low density ices (i.e., $\mathrm{H}_{2} \mathrm{O}, \mathrm{NH}_{3}$ ), are essentially spherical, ${ }^{1}$ and have diameters which range from just under $400 \mathrm{~km}$ to nearly 1600 km (Smith et al., 1981, 1982, 1986). Not surprisingly, all of these objects exhibit impact craters. On many of the middle-sized, icy satellites, very large impact features

\footnotetext{
* Corresponding author. Fax: (650)-604-6779.

E-mail address: jeff.moore@nasa.gov (J.M. Moore).

${ }^{1}$ More specifically, triaxial ellipsoids in tidal and rotational equilibrium.
}

with diameters reaching a substantial fraction of the satellite diameter are observed (Fig. 1). It is these very large impact features, and the effects these features have had on their targets, that are the subjects of this study.

The largest impact features on a satellite usually have a major effect on its geologic history. On terrestrial planets, very large impacts established widespread stratigraphic horizons, long-lasting structural imprints, and sometimes sites of subsequent volcanic activity (Spudis, 1982; Wilhelms, 1987). Large impacts have substantially altered surrounding pre-impact surface morphology through ejecta emplacement (e.g., Gilbert, 1893; Boyce et al., 1974) and seismic shock (Schultz and Gault, 1975). On the large, icy galilean satellites the regions surrounding very large impact features have undergone tectonic, and possibly cryovolcanic, post-impact modification (e.g., McKinnon and Melosh, 1980). Galileo-era studies of large impact features on galilean satellites have focused on the prompt forma- 


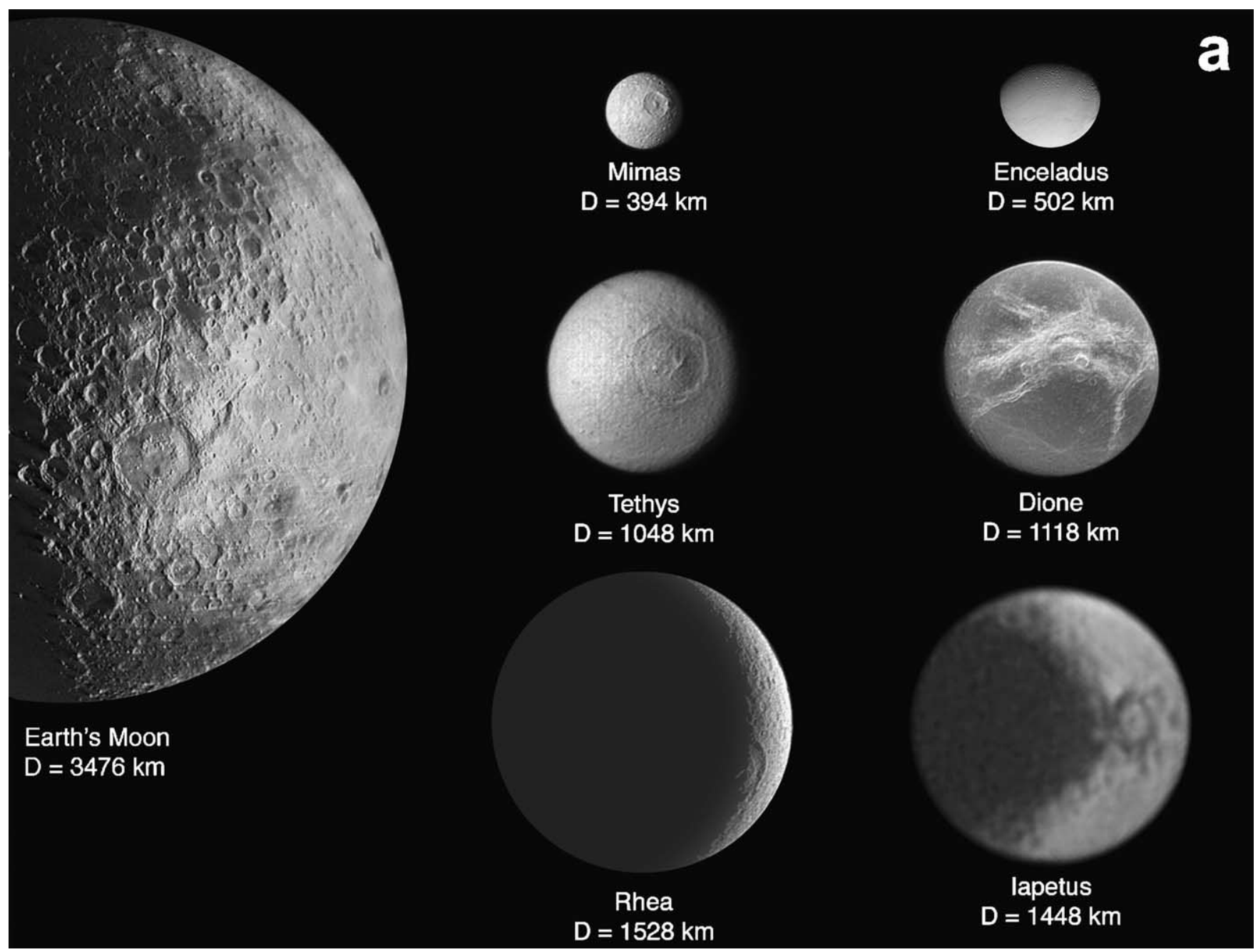

Fig. 1. The middle-sized, icy satellites, shown to a common scale. The Earth's Moon is included for comparison. Individual satellite images were selected to portray the largest impact feature observed on a given body. Radii are from Burns (1986). (a) The saturnian middle-sized satellites. North is roughly toward the top of the figure. The peak-ring basin, Schrödinger (diameter $320 \mathrm{~km}$ ), lies in the middle of this view of the Earth's Moon. Schrödinger is comparable in size and, to some extent, appearance to some of the large impact features on the icy satellites (e.g., Odysseus on Tethys, and Tirawa on Rhea). The images on this montage are: Moon, Lunar Orbiter IV-8-M; Mimas, Voyager FDS 34933.50; Enceladus, Voyager FDS 44000.42; Tethys, Voyager FDS 43975.07; Dione, Voyager FDS 34933.38; Rhea, Voyager FDS 34963.08; Iapetus, Voyager FDS 34951.56. (b) The uranian middle-sized satellites. For these bodies, north is roughly toward the right of the figure. The large basin at the top of the lunar image is Orientale. The images used in this montage are: Moon, Lunar Orbiter IV-180-M; Miranda, Voyager FDS 26844.13; Ariel, JPL Voyager mosaic P-29520; Umbriel, Voyager FDS 26840.04; Titania, Voyager FDS 26843.13; Oberon, Voyager FDS 26836.23.

tion of non-crater morphologies such as palimpsests (see review in Schenk et al., 2004), the post-impact modification of crater topography (e.g., Moore et al., 1998, 2001; Schenk, 2002), and possible antipodal focusing of impactgenerated seismic energy (see Section E in Moore et al., 2004). Previous studies of large impact features on middlesized icy satellites have considered the role of antipodal focusing of impact-generated seismic energy using simple modeling (e.g., Watts et al., 1991) and the role of postimpact topographic modification due to viscous relaxation (e.g., Schenk, 1989; Thomas and Squyres, 1988; McKinnon and Benner, 1989).

In this report we review, on the eve of Cassini's arrival in the saturnian system, the state of understanding of the role large impact features have played in the histories of middlesized icy satellites. We describe large impact features and their related landforms as they were seen in Voyager data. We interpret and speculate about these features, which we use as a basis to evaluate the effects of very large impacts on the global geology of the target satellites. We discuss the relative roles of ejecta deposition and secondary cratering, and the distribution of seismic energy across a given target satellite. We evaluate observational constraints in the context of new modeling reported in Bruesch and Asphaug (2004). We conclude by proposing both tests for our interpretations and opportunities to resolve ambiguities that could be conducted by the upcoming Cassini mission to the Saturn system. 


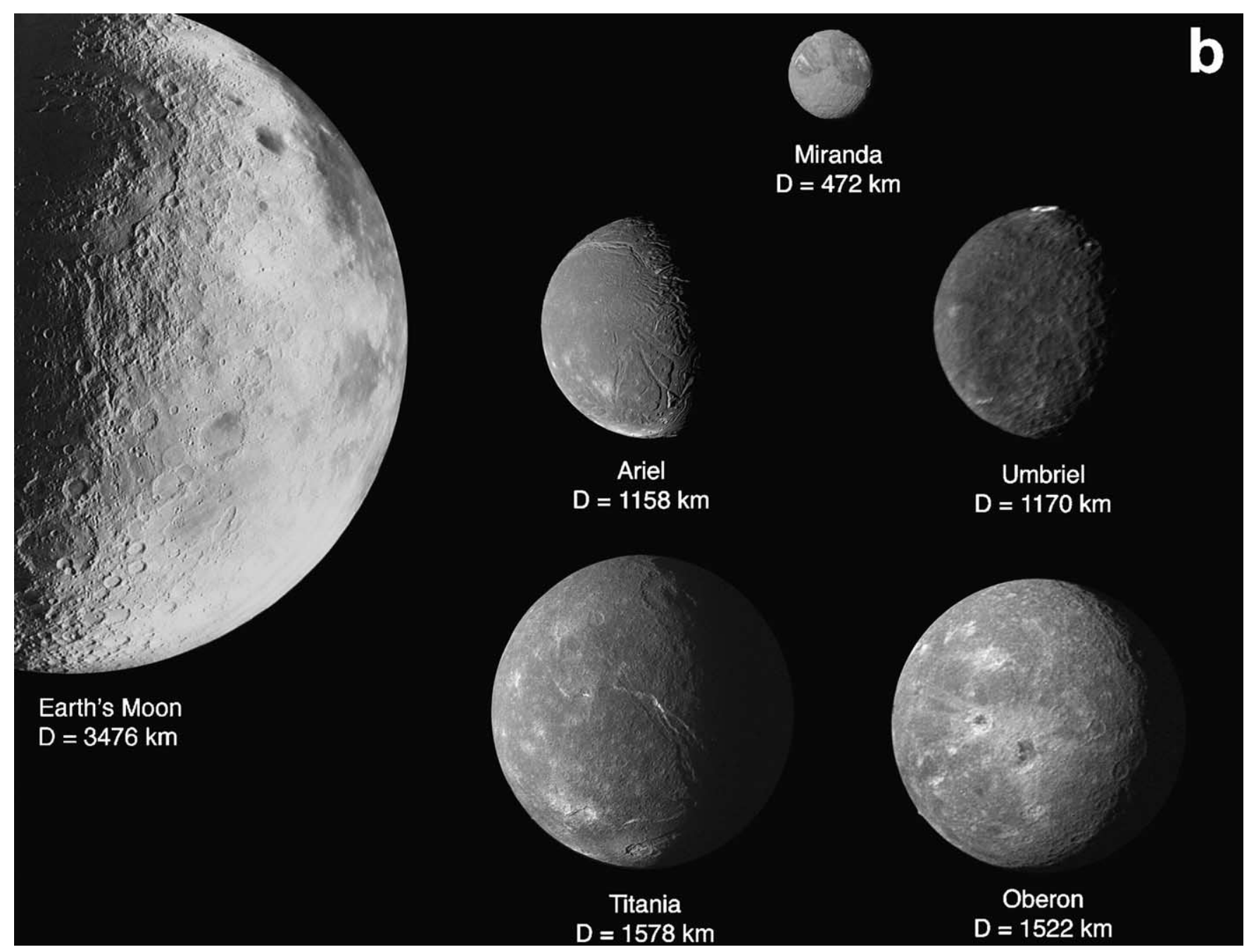

Fig. 1. Continued.

\section{Observations and interpretations}

\subsection{Topographic determination technique}

Prior to this study, topographic mapping of the middlesized icy satellites has been limited to limb profiles using a technique developed by Thomas (e.g., Thomas, 1988; Dermott and Thomas, 1988; Thomas and Dermott, 1991) and limited photoclinometric line profiles of selected features (Schenk, 1989). Our new topographic mapping of these satellites is based on two methods, stereo image analysis and 2-dimensional photoclinometry of low-sun regions, which allows us to produce digital elevation models (DEMs) of large surface areas. Stereo analysis is based on an automated scene recognition algorithm successfully applied to the galilean satellites (i.e., Schenk and Bulmer, 1998; Schenk, 2002). Areal coverage of stereo DEMs is limited by the rapid nature of the Voyager flybys. Rhea and Dione have the best stereo coverage, with $\sim 25 \%$ of the surface mappable at better than $300 \mathrm{~m}$ vertical resolution and better than $5 \mathrm{~km}$ horizontal resolution on both satellites. Some stereo coverage was obtained for Tethys, Enceladus, and Mimas, but these satellites all revolve more rapidly than Rhea and Dione and the large changes in solar illumination azimuth during the Voyager imaging sequences restrict useful stereo coverage. We note that for large deep craters, even the lowresolution stereo coverage for these satellites can be useful. Iapetus was viewed at no better than $7 \mathrm{~km} \mathrm{pixel}^{-1}$ resolution by Voyager and no useful stereo topography over large craters can be derived.

Photoclinometry greatly extends topographic coverage, with the proviso that long-wavelength topographic variations are suspect using this technique, unless controlled by coincident stereo coverage. We employed a new photoclinometry algorithm developed by one of us (PMS) for rapid 2-dimensional photoclinometric mapping that includes modeling of local albedo changes where possible (P.M. Schenk, Topography of Europa, in preparation). Photoclinometric mapping is generally limited to areas within $30^{\circ}$ of the local terminator.

A necessary precursor to topographic mapping using imaging is the existence of an accurate coordinate control 
network for the Voyager images. Extensive effort by one of us (PMS) was made to adjust the control nets for all relevant satellite images using USGS ISIS software. In order to do so successfully, it was desirable to close the network over $360^{\circ}$ of longitude despite the fact that there was usually a factor of 20 or more difference in the resolution from the first to the last images used. Based on these new networks we have constructed new global mosaics for each satellite using the best available images for all surface areas and used here as our base maps. These new control nets may be of use to update Cassini targeting and are available by request. Our new data were used here to update topographic measurements of large impact features, superseding those made over a decade ago (Schenk, 1989).

\subsection{Mimas}

Mimas (average diameter $400 \mathrm{~km}$; Dermott and Thomas, 1988 ) is the smallest and innermost of the middle-sized, icy satellites of Saturn. It was best imaged by Voyager 1, which provided coverage primarily of the southern hemisphere. Its surface is dominated by craters, apparently without significant endogenic modification.

The most conspicuous feature on Mimas is the $135 \mathrm{~km}$ diameter crater Herschel $\left(0^{\circ} \mathrm{N}, 111^{\circ} \mathrm{W}\right)$, which is nearly one-third the diameter of the satellite (Figs. 1a, 2a, 3a, and 4). Herschel has the form of a classic central-peak crater and appears to have not undergone detectable morphologic degradation at the resolution of the best Voyager images $\left(4 \mathrm{~km} \mathrm{pixel}^{-1}\right)$. Unlike central-peak craters of this size on the Moon, Herschel has no apparent slump block terraces around its interior rim, though this may be a resolution issue. Smith et al. (1981) estimated, based on shadow measurements, that Herschel's walls have an average height of $5 \mathrm{~km}$, and parts of the floor may be $\sim 10 \mathrm{~km}$ deep (Schenk, 1989). They determined that the central peak, which is 20 by $30 \mathrm{~km}$ at its base, rises $\sim 6 \mathrm{~km}$ from the crater floor.

We confirm that Herschel is $\sim 10-12 \mathrm{~km}$ deep. The lunar equivalent crater would be $\sim 4.5 \mathrm{~km}$ deep, according to the empirical expression:

$H=1.044 D_{\mathrm{f}}^{0.301}$,

where $H$ and $D_{\mathrm{f}}$ are crater depth and rim diameter, in km (Pike, 1980). Herschel's rim height above its surroundings is 1 to $2.5 \mathrm{~km}$, which is comparable to the lunar equivalent crater rim height of $1.6 \mathrm{~km}$, according to the empirical expression:

$h=0.236 D_{\mathrm{f}}^{0.399}$,

where $h$ is rim height, in $\mathrm{km}$ (Pike, 1980). We determined that Herschel's central peak rises $\sim 6-8 \mathrm{~km}$ from the crater floor (Fig. 3a). The height of the central peak above the crater floor for a $130 \mathrm{~km}$ lunar crater is $\sim 2-3 \mathrm{~km}$ (Hale and Grieve, 1982), with a basal width of $\sim 30 \mathrm{~km}$ (Hale and Head, 1979). The greater relief within Herschel with respect to a $130 \mathrm{~km}$-diameter lunar crater is most likely a consequence of Mimas's much lower gravity ( $\sim 0.025$ that of the Moon), as has been suggested by Smith et al. (1981). Schenk (1989) further suggested that the mechanical reason for this difference is that terrace formation, which widens and shallows lunar craters, is not as prominent a process on the middlesized icy satellites (cf., Chapman and McKinnon, 1986; McKinnon et al., 1991). Hopefully, Cassini will be able to look for terraces on Herschel and other large craters. At the end of the paper we provide candidate observations for Cassini to resolve these and other ambiguities.

Low resolution images indicate that topography is softened or mantled near the rim of Herschel in a manner consistent with deposition of continuous ejecta, but resolution precludes explicit recognition of such a deposit. However, ejecta scaling developed by Housen et al. (1983) and discussed in Veverka et al. (1986) indicates that the amount of ejecta that would reach Mimas escape velocity from the Herschel impact event would be $\sim 20-25 \%$, leaving most of the ejecta to fall back on the satellite. ${ }^{2}$ Subsequent analysis of ejecta distribution in large impact craters on low-gravity bodies by Asphaug and Melosh (1993) support this result.

Linear-to-arcuate, sub-parallel troughs on Mimas (Fig. 4) are each typically $\sim 10 \mathrm{~km}$ wide and individual troughs are seen to extend across $\sim 120^{\circ}(\sim 400 \mathrm{~km})$ of its globe. These troughs are a significant landform in some regions of the satellite, and are the only non-crater landform recognized in Voyager images. The lack of secondary crater morphologies, such as strings of pits with raised rims, suggests that these are fractures within Mimas. These troughs are hypothesized to have formed as global-scale fractures during the Herschel impact event (McKinnon, 1985; Schenk, 1985) as a case of incipient global breakup. This hypothesis is similar to one that relates the formation of troughs or grooves on the martian satellite Phobos to its large crater Stickney (Thomas, 1987). It should be noted, however, that while many of the more prominent troughs on Mimas (Fig. 4) radiate from Herschel the way grooves do on Phobos from Stickney, some do not. Whether troughs on Mimas are pre-existing fractures widened by impact-induced seismic energy during Herschel formation (Schenk, 1985) or new fractures formed during the cratering event is unknown. If pre-existing fractures, they may perhaps have been formed by body stresses associated with tidal evolution (e.g., Soter and Harris, 1977; Melosh, 1980), although this has not been evaluated due to the incompleteness of coverage.

\subsection{Tethys}

Tethys (1060-km average diameter; Thomas and Dermott, 1991) received the most complete areal coverage (at

\footnotetext{
${ }^{2}$ Veverka et al. (1986) derive an approximate expression for the mass fraction escaping for gravity-regime impacts into non-porous "targets," $f_{\text {esc }} \sim 0.6\left(D_{\mathrm{f}} / D_{\text {sat }}\right)^{0.85}$, where $D_{\text {sat }}$ is the satellite diameter. For porous targets, which may apply to Mimas (Eluszkiewicz, 1990), $f_{\mathrm{esc}} \sim$ $0.4\left(D_{\mathrm{f}} / D_{\text {sat }}\right)^{0.6}$.
} 


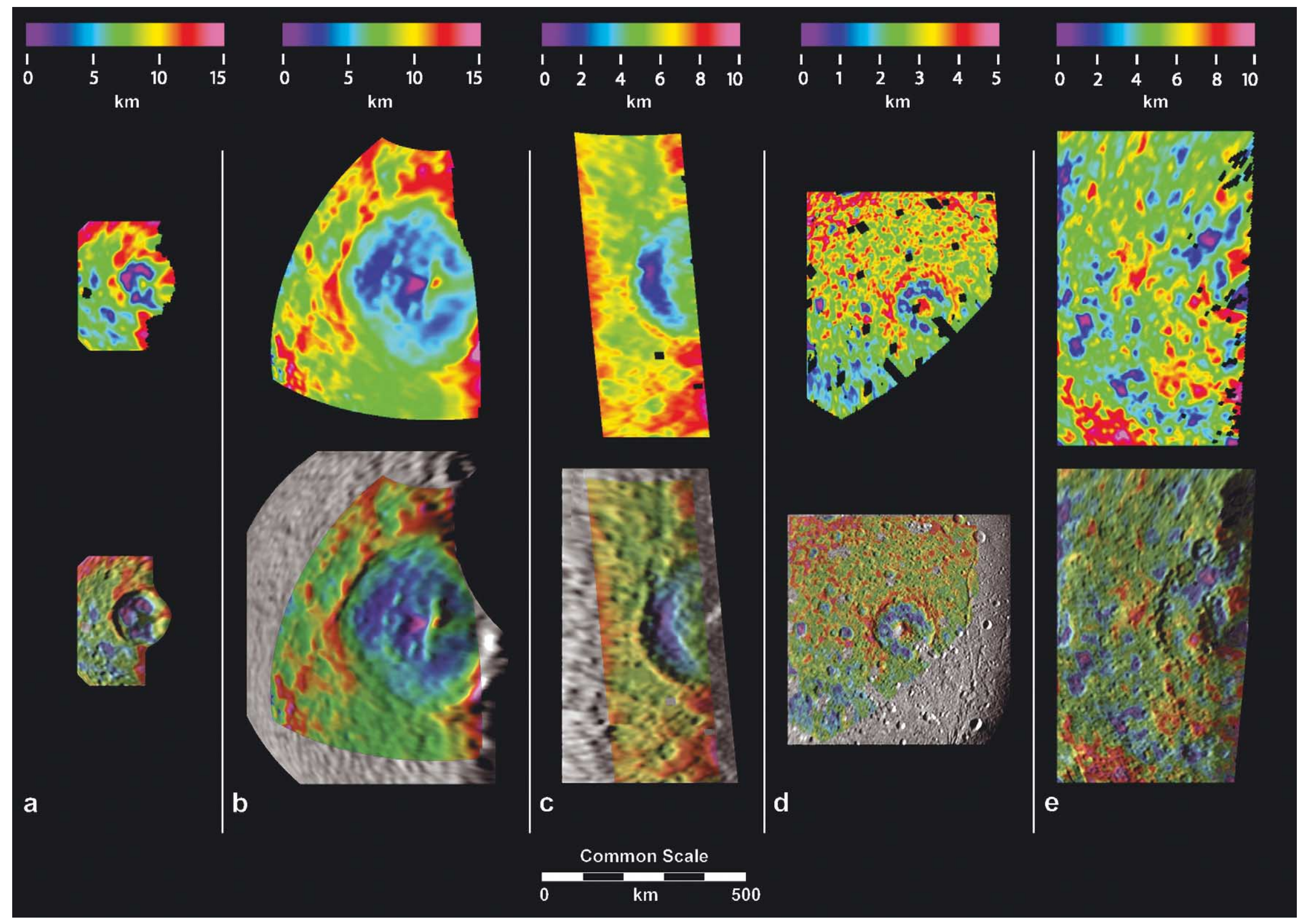

Fig. 2. Five map-projected Voyager images of some of the impact features discussed in the text "colorized" with Digital Elevation Models (DEMs) of these features. The DEMs are also shown separately with

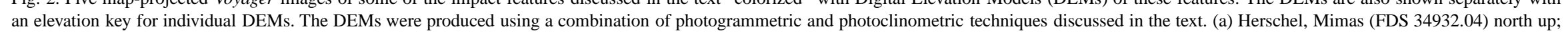
(b) Odysseus, Tethys (FDS 43980.27) north up; (c) Tirawa, Rhea (FDS 34963.08) south up; (d) Aeneas, Dione (FDS 34948.22) south up; (e) Gertrude, Titania (FDS 26843.13) west up. All images have been transformed to feature-centered, Lambertian equal-area projections and all to a common plan scale. 


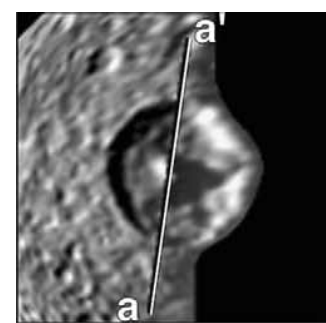

Herschel

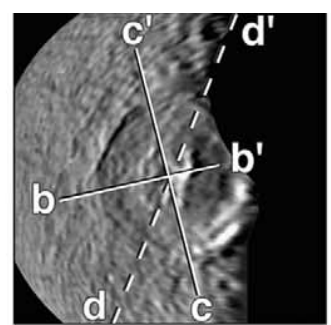

Odysseus

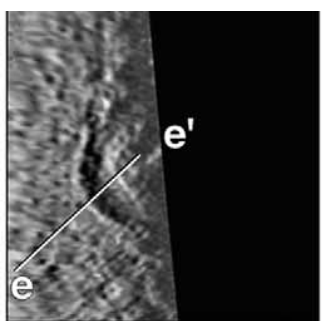

Tirawa

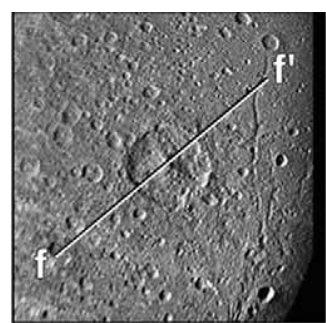

Aeneas

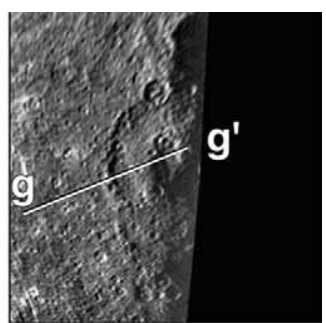

Gertrude
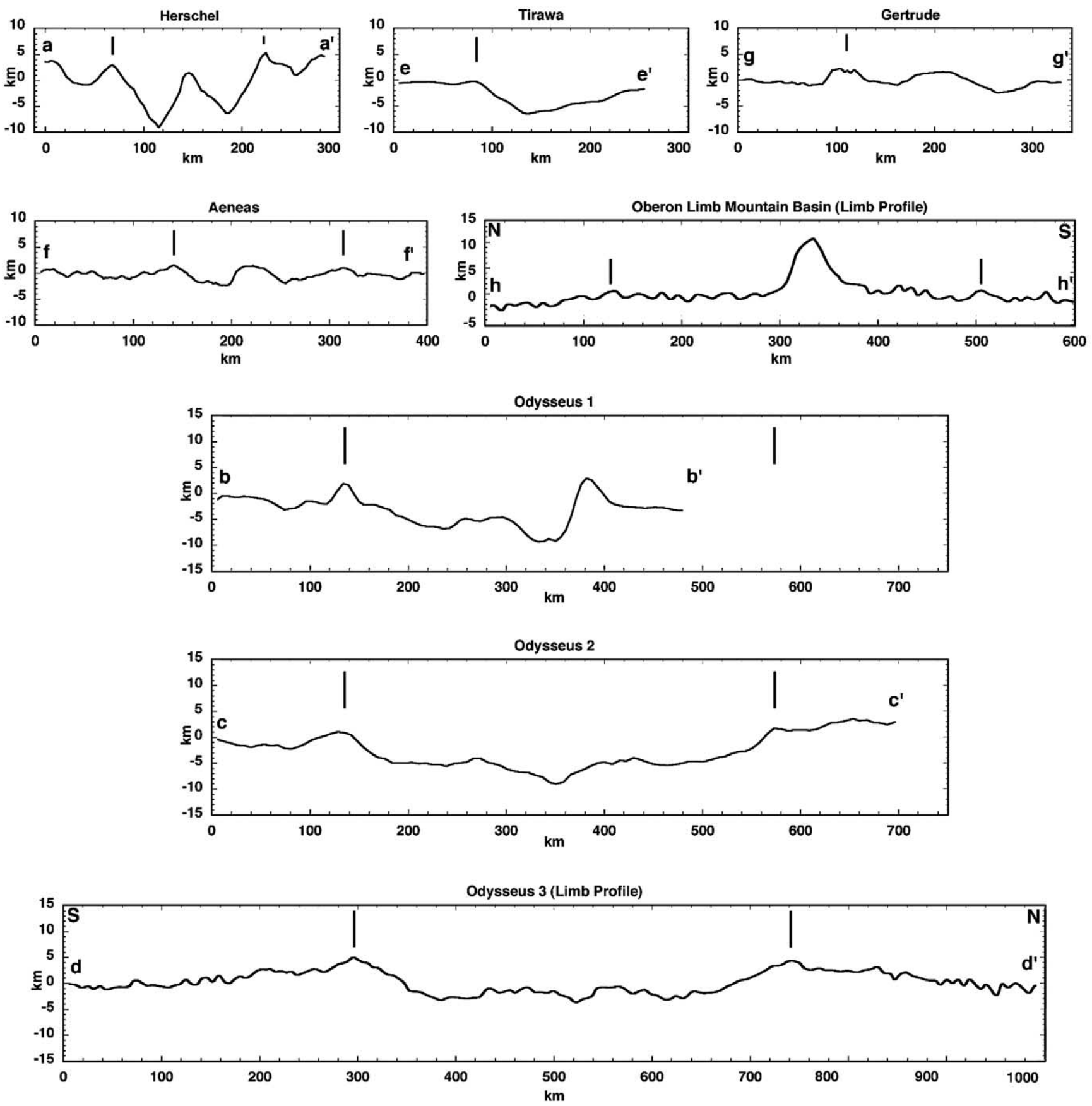

Fig. 3. Topographic profiles of the large impact features discussed in the text. The vertical exaggeration is $20: 1$. The superimposed lines on the inset images show where the topographic profiles were determined. The dashed line on the Odysseus inset shows the approximate trace of limb profile (d). Profiles (a)-(c), (e)-(g) were derived from the DEMs shown in Fig. 4. Profiles (d) and (h) were derived from the limbs on Voyager images (Oberon. FDS 26836.23; Tethys. FDS 34937.10) using the limb fitting technique discussed in Dermott and Thomas (1988) (courtesy P.C. Thomas) 


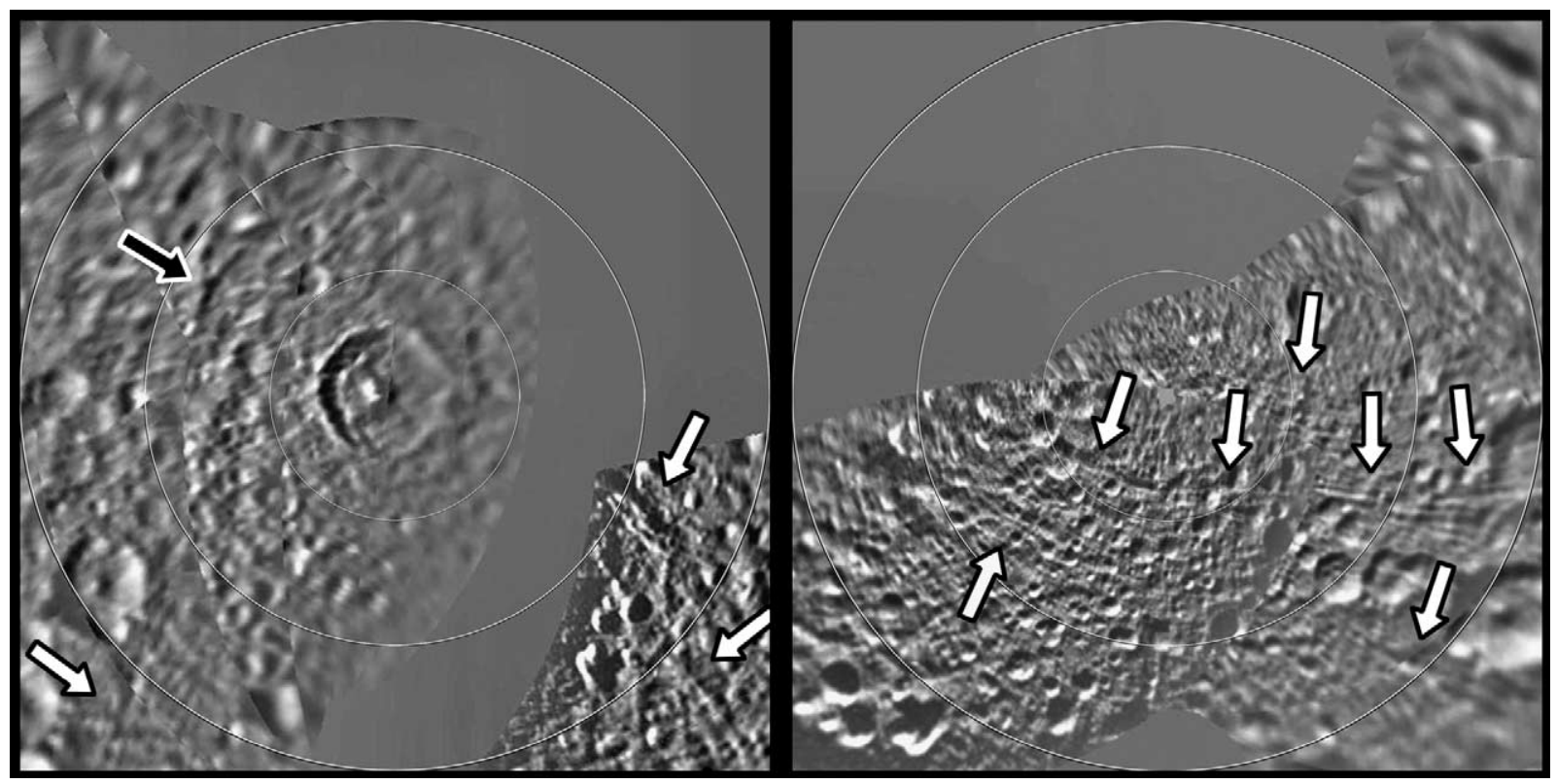

Fig. 4. Mosaics of Voyager images of Mimas. The two views are polar stereographic projections centered on (left) the sub-Herschel hemisphere and (right) the anti-Herschel hemisphere, respectively. Arrows point to prominent linear troughs. The circles inscribe points $30^{\circ}$, $60^{\circ}$, and $90^{\circ}$ from the projection center (for scale, $30^{\circ}$ equals $\sim 100 \mathrm{~km}$ in ground distance). North is up. Gray areas denote lack of useful image coverage.

resolutions from $\sim 20$ to $3 \mathrm{~km}_{\text {pixel }}{ }^{-1}$ ) of any saturnian satellite by the Voyager spacecraft. There are two major surface types on Tethys: an undulatory, hilly and cratered terrain dominated by large ( $>40 \mathrm{~km}$ diameter) craters, and a smaller plains region displaying lower relief and a mostly small ( $<20 \mathrm{~km}$ diameter) crater population (Smith et al., 1982), where seen in the higher resolution images.

Centered on $30^{\circ} \mathrm{N}, 130^{\circ} \mathrm{W}$, Odysseus (Figs. 1a and 5) is the largest impact feature on Tethys, and perhaps the largest such feature on any saturnian satellite. This feature, Odysseus, has a diameter of $400 \mathrm{~km}$ in chord distance, about $40 \%$ the diameter of the satellite. However, Odysseus's floor matches the spherical curvature of the satellite. The feature is $\sim 450 \mathrm{~km}$ from rim to rim as measured along Tethys' circumference. North-south limb profiles across Odysseus and it surroundings generated by P.C. Thomas, using his limb-fitting technique (Thomas, 1988; Thomas and Dermott, 1991), show that the exterior rim flank width is $\sim 200 \mathrm{~km}$. Over this distance the surface rises with increasing slope until it is $\sim 5 \mathrm{~km}$ above the mean satellite radius at the rim crest (Figs. 3b-3d). The interior rim of Odysseus is composed of arcuate scarps, which in some places are doubled, though the resolution of Voyager images does not permit identification of this ridge-doubling as terracing. The relief of Odysseus drops from the rim at a more-or-less constant slope $\left(5^{\circ}\right)$ for $\sim 100 \mathrm{~km}$ in the north-south direction until the floor is reached at $\sim 8 \mathrm{~km}$ below the crest, but only $3 \mathrm{~km}$ below the mean satellite radius. Our stereocontrolled photoclinometric DEM (Fig. 2b) suggests rimto-floor relief of 6-9 km, consistent with the limb profiles.

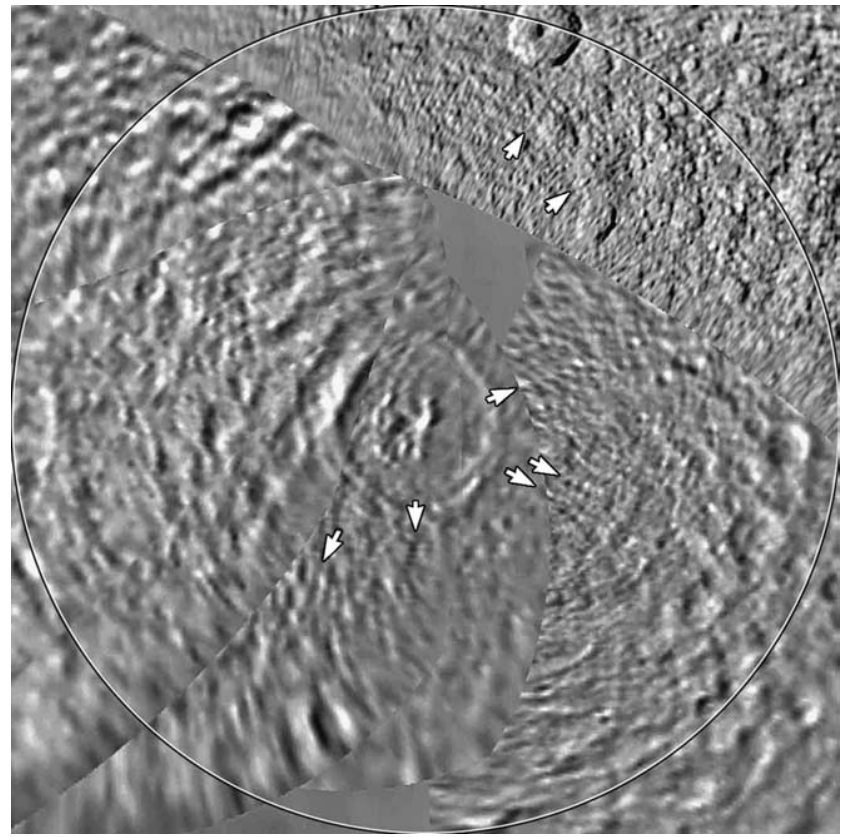

Fig. 5. Polar stereographic projection of a Voyager Tethys mosaic, centered on the Odysseus impact feature. The white arrows point to lineaments radial to Odysseus. The circle inscribes points $90^{\circ}$ from Odysseus (for scale, $90^{\circ}$ equals $825 \mathrm{~km}$ in ground distance).

Our topographic mapping (Figs. $2 \mathrm{~b}$ and $3 \mathrm{~b}-3 \mathrm{~d}$ ) suggests that the central $200 \mathrm{~km}$ of Odysseus consists of a central pit 2-4 km deep surrounded by an annular ring of massifs or ridges of variable height (the lack of relief in the limb profile could be a projection effect). This complex may be elevated $2-3 \mathrm{~km}$ above the surrounding basin floor. Odysseus may have some elements in common with cen- 
tral pit craters on Ganymede (e.g., Moore and Malin, 1988; Schenk, 1991) or lunar, martian, and mercurian peak-ring basins. Peak-ring basins are hypothesized to form at the collapse stage during the formation of very large craters. The rebounding central uplift, which is thought to form central peaks in smaller complex craters, collapses leaving a central ring of peaks (e.g., Melosh, 1989, pp. 126-162; Collins et al., 2002). Two important factors that determine whether a very large crater will form a peak ring are the mechanical properties of the material within the crater during the collapse stage and the role of target gravity (e.g., Melosh, 1989). Voyager image quality is insufficient to evaluate the veracity of this comparison. The recognition of peak-ring basin analogs, as opposed to central pits, on middle-sized icy satellites would serve to test among various hypotheses for the role of gravity in their formation and the nature of the target material in which they form, especially in light of their absence on the icy galilean satellites.

Given the high albedo $(\sim 0.8)$ and low-resolution images available for this area of Tethys, the undetectability in Voyager images of obvious young bright ejecta deposits is not surprising. However, according to the ejecta scaling of Veverka et al. (1986), discussed above, not more than $\sim 25 \%$ of the ejecta from the Odysseus impact event would escape Tethys.

There are several trough-like features several hundred kilometers long and 10-20 km wide, which radiate away from Odysseus (Fig. 5). Some of these troughs appear to be composed of chains of coalescing pits or craters. Though the limitations of resolution do not permit an unequivocal identification, the arrangement and size of the troughs grossly resemble large grooves created by secondary impacts which radiate from lunar (i.e., Schrödinger) and mercurian (i.e., Strindberg and Ahmad Baba) peak-ring basins. An alternative possibility is that the troughs may be radial fractures induced by extensional stresses associated with (deeper) viscous relaxation and rise toward an equipotential surface of impact-feature topography (Melosh, 1976). Hopefully, Cassini will obtain images at sufficient resolution to determine whether these troughs have features that support a secondary impact interpretation (e.g., raised rims, associated outer rim herringbone patterns) or features consistent with tectonic extension (e.g., inward facing parallel fault scarps).

The region antipodal to the center of Odysseus is in a plains unit (Fig. 6). The gravitational binding energy of Tethys is more than one or two orders of magnitude greater than the kinetic energy imparted to the satellite by the Odysseus impact depending on the impact scaling used (see Section 3). The possibility that the plains terrain is the direct consequence of seismic energy focused at the impact point antipodal cannot be completely ruled out. The effect of seismic shaking (and subsequent ejecta mantling) is to degrade pre-existing landforms. On the Moon and Mercury (Fig. 7), pre-existing landforms in regions located antipodal to very large impact features are overprinted and modified with rugged, equidimensional hills or mounds and nar-

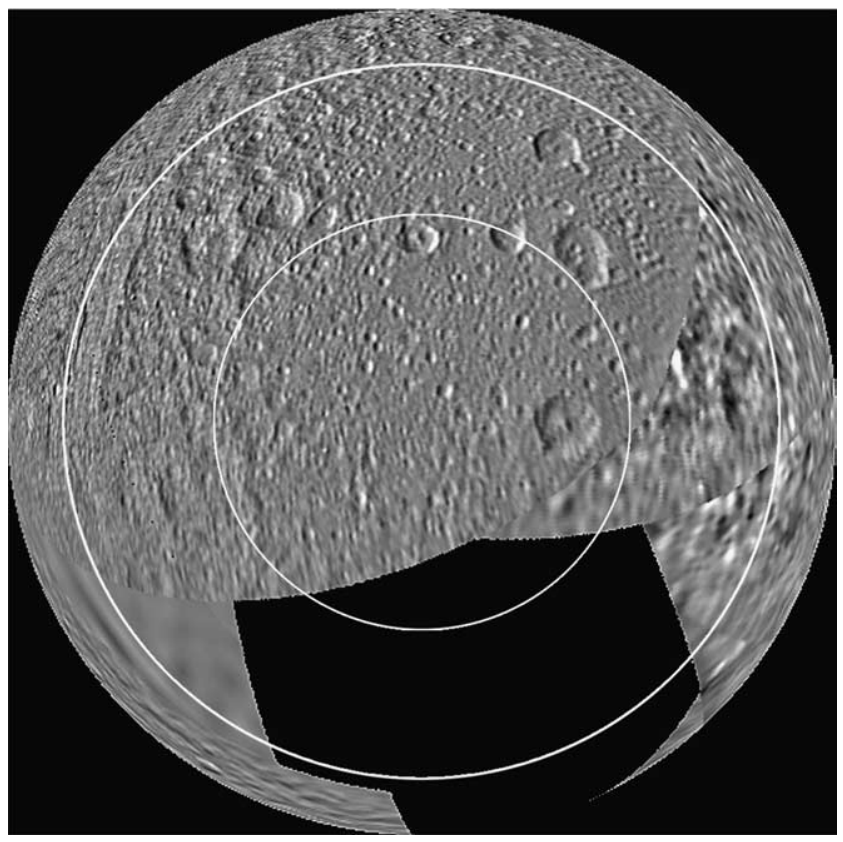

Fig. 6. Lambertian equal-area projection of a Voyager Tethys mosaic of the anti-Odysseus hemisphere centered on the Odysseus antipode $\left(30^{\circ} \mathrm{S}\right.$, $310^{\circ} \mathrm{W}$ ). The circles inscribe points $30^{\circ}$ and $60^{\circ}$ from the Odysseus antipode (for scale, $30^{\circ}$ equals $275 \mathrm{~km}$ in ground distance).

row linear troughs or grooves (Schultz and Gault, 1975; Stuart-Alexander, 1978; Trask and Dzurisin, 1984). In extreme cases, conceivably, a seismically shaken surface could be reduced to a level plain.

Resolution quality of the Voyager images of Odysseus prevents a determination of the impact feature's age with respect to the plains by either stratigraphic means or by relative crater density. However, as has been noted by Moore and Ahern (1983), the plains boundary, where seen in a highresolution image (Fig. 6), is relatively abrupt and even scarplike in several places. Figure 6 also shows that the plains unit is not centered on the Odysseus antipode but extends for at least $60^{\circ}$ northeast of that point. If the plains were formed by seismic shaking, there would probably be a significant transition zone between that unit and the hilly and cratered terrain (i.e., a blocky, rugged zone similar to Mercury's hilly and lineated terrain). As this appears not the case, a reasonable interpretation is that the plains unit was formed by an episode of endogenic extrusion subsequent to the formation of Odysseus. These extrusive flows were stopped in the west by scarp-like or rising topography. Moore and Ahern (1983) hypothesized, as was reiterated by Watts et al. (1991), that the Odysseus impact created lines of weakness in the antipodal lithosphere providing an access to the surface for the extrusive material.

Ithaca Chasma (Fig. 8), an enormous trough system extending at least $270^{\circ}$ around Tethys, is narrowly confined to a zone which lies roughly along a great circle (Fig. 8b) that is concentric with Odysseus (Smith et al., 1982; Moore and Ahern, 1983). Ithaca Chasma was described by Smith et al. (1981) as a branching, terraced trough with an estimated av- 


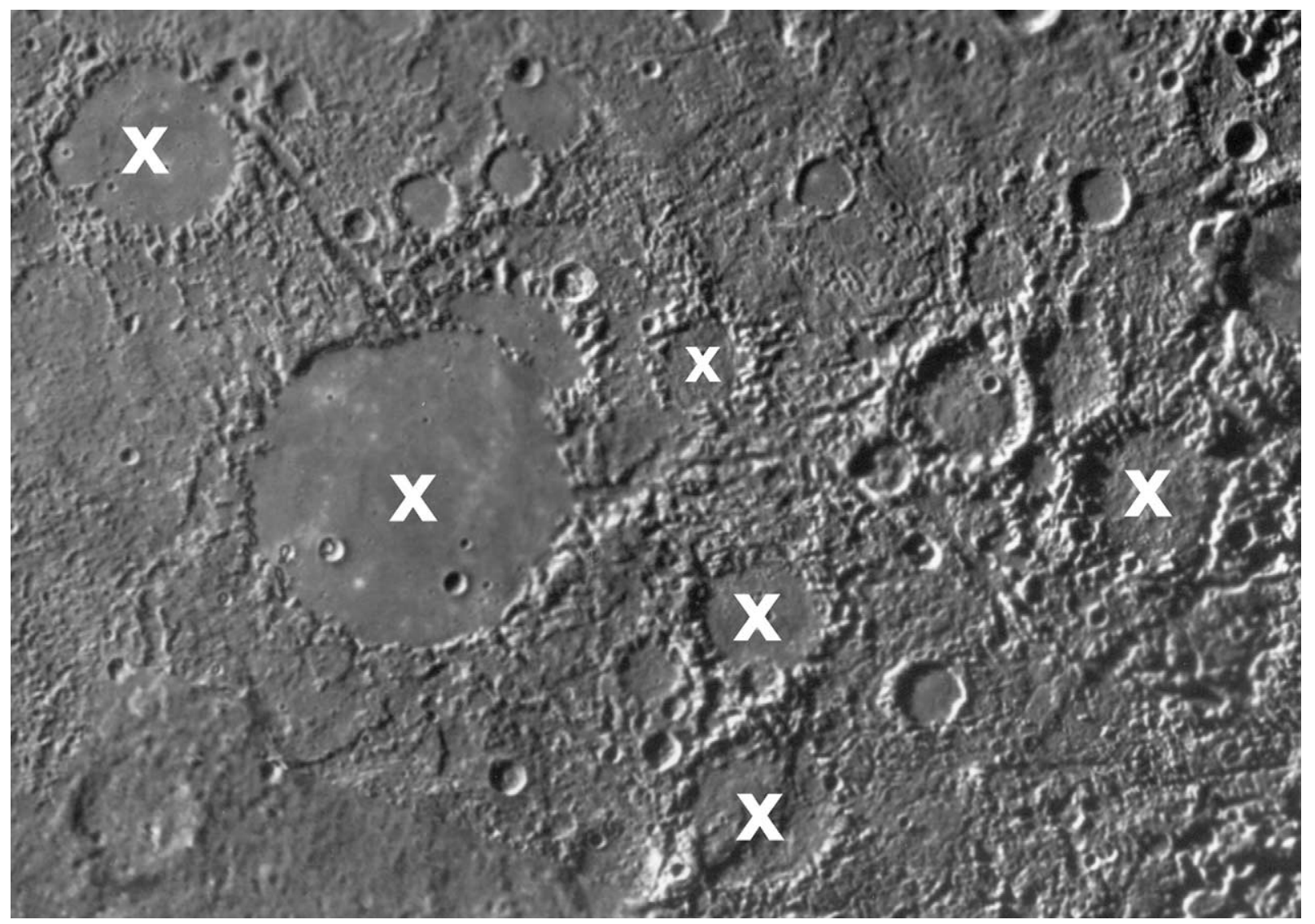

Fig. 7. Hilly and lineated terrain, centered $\sim 30^{\circ} \mathrm{S}, 23^{\circ} \mathrm{W}$, antipodal to the Caloris impact basin on Mercury. Pre-Caloris intercrater areas (see especially the lower right of the figure) and crater rims (examples indicated with " $\mathrm{X}$ ") have been broken into troughs and equidimensional hills presumably as a result of violent faulting, mass-wasting, and degradation initiated by seismic energy focused at this locality from the Caloris impact. North is roughly toward the top of the figure. Lighting is from the left. The scene is $\sim 675 \mathrm{~km}$ across (portion of Mercury Map Quadrangle H-11 photomosaic).

erage depth of $3 \mathrm{~km}$, a width varying from only a few tens of kilometers to over $100 \mathrm{~km}$, and, in places, a raised rim as high as $0.5 \mathrm{~km}$ above its surroundings. Over much of its length, the walls of Ithaca Chasma are complex, composed of multiple sub-parallel scarps. Smith et al. (1981) suggested that Ithaca Chasma was formed by freeze-expansion of Tethys's interior. They noted that if Tethys was once a sphere of liquid water covered by a thin solid ice crust, freezing of the interior would have produced expansion of the surface comparable to the area of the chasm $(\sim 10 \%$ of total satellite surface area). This hypothesis failed to explain why the chasm occurs only within a narrow zone, and suffers from the extreme geophysical difficulty of accounting for the energy release needed to create a molten interior. Expansion of the satellite's interior should have caused fracturing to occur over the entire surface in order to effectively relieve stresses in a rigid crust (Moore and Ahern, 1983). In addition, Tethys' low density of $\approx 1000 \mathrm{~kg} \mathrm{~m}^{-3}$, based on celestial mechanics solutions (Campbell and Anderson, 1989; Dourneau and Baratchart, 1999), implies a low rock mass fraction ( $\sim 10 \%$ if non-porous) and very modest radiogenic heating.

The geometric relationships between Odysseus and Ithaca Chasma prompted Moore and Ahern (1983) to suggest the trough system was an immediate manifestation of the impact event, perhaps due to a damped, whole-body oscillation of the satellite. Indeed, our new global mosaic (Fig. 8) shows that Ithaca Chasma is offset by no more than $\sim 20^{\circ}$ from the great circle inscribing Odysseus. Based on the 3-km-deep topography of Odysseus, Schenk (1989) also suggested that Odysseus had not undergone substantial relaxation, and that Ithaca Chasma was the equivalent of a ring graben formed during the impact event by prompt floor collapse involving a large portion of the interior. ${ }^{3}$ In this study we explore the possibility that Ithaca Chasma was promptly formed as a consequence of the Odysseus impact.

Measurement of crater densities inside Ithaca Chasma using Voyager images led Plescia and Boyce (1983) to conclude that the trough system is perhaps the youngest feature on Tethys. If Odysseus proves to be older that Ithaca Chasma, this would support the alternative hypothesis that the formation of Ithaca Chasma manifested itself by stresses induced during relaxation over an extended period follow-

\footnotetext{
${ }^{3}$ Odysseus was judged by Schenk (1989) to be no deeper than a crater of its size should be, based on extrapolation of smaller, complex crater depths on the satellite.
} 


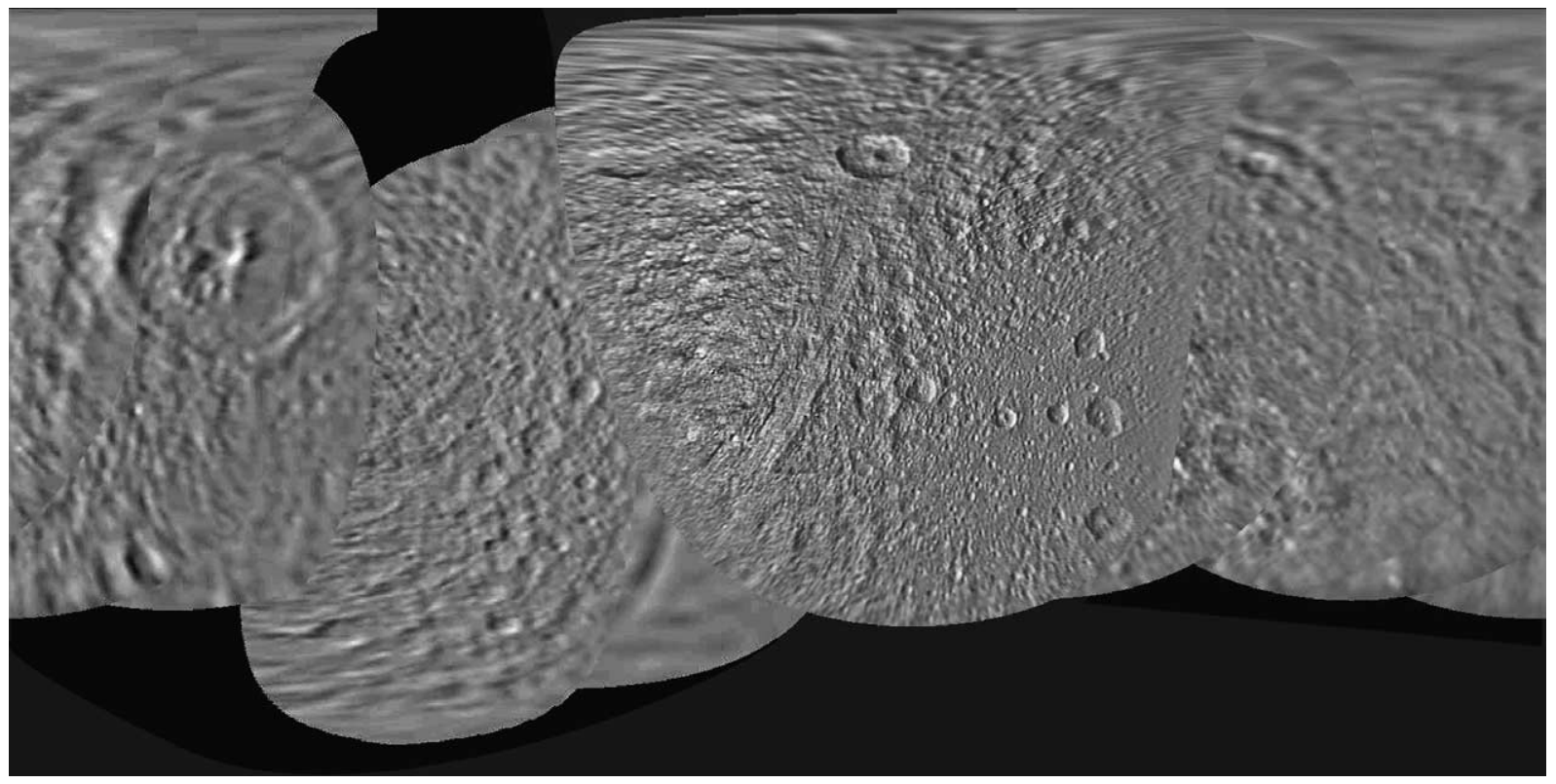

(a)

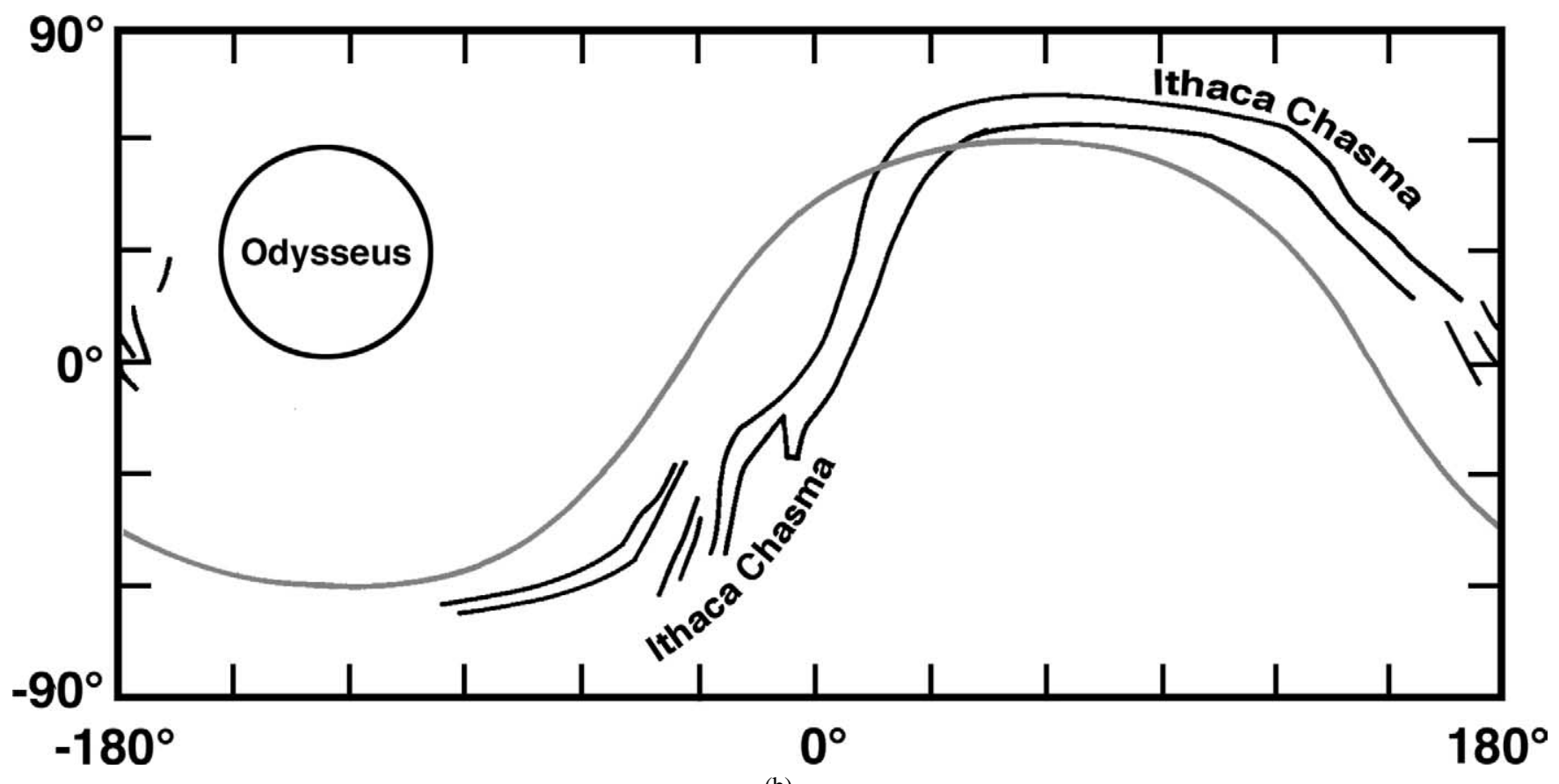

(b)

Fig. 8. (a) A mosaic of Voyager images of Tethys in cylindrical projection. (b) A trace of Ithaca Chasma and a great circle centered on Odysseus showing a possible relationship between these two features on Tethys.

ing the impact (Thomas and Squyres, 1988; McKinnon and Benner, 1989). High-resolution crater counts of the floors of Odysseus and Ithaca Chasma will be key to determining the relative ages of these two features. However, we note that the formation of simple graben would not necessarily reset the cratering history of the downdropped blocks within the graben. A careful mapping of the stratigraphic history will be needed to establish the relative ages of these two features.

\subsection{Rhea}

Rhea, at $1528 \mathrm{~km}$ in diameter, is Saturn's second largest satellite. With a density of $\sim 1250 \mathrm{~kg} \mathrm{~m}^{-3}$ compared with $\sim 1000 \mathrm{~kg} \mathrm{~m}^{-3}$ for Tethys (Yoder, 1995), Rhea is also more silicate-rich. The largest unequivocal impact feature on Rhea observed in Voyager data is Tirawa (centered $36^{\circ} \mathrm{N}$, $150^{\circ} \mathrm{W}, 350 \mathrm{~km}$ in diameter). There is a single moderate resolution $\left(5 \mathrm{~km} \mathrm{pixel}^{-1}\right)$ view of Tirawa seen at low 
illumination and high $\left(155^{\circ}\right)$ phase angle (Figs. 1a, 2c, and $3 \mathrm{e})$. Additionally, there are a few low resolution $(<$ $25 \mathrm{~km}$ pixel $\left.^{-1}\right)$, low phase angle $\left(<15^{\circ}\right)$ views which show an annulus $\sim 450 \mathrm{~km}$ in diameter that is slightly higher in albedo than its surroundings. The outer rim corresponds with the inner edge of the bright annulus seen at low resolution. This annulus may be part of a continuous ejecta deposit.

Photoclinometrically-derived topography of Tirawa (Figs. 2c and 3e) indicates that the exterior rim begins rising $\sim 60 \mathrm{~km}$ away from the crest until it reaches a height of $\sim 1.3 \mathrm{~km}$ above the exterior surroundings. The relief from the rim crest to the floor is at least $6 \mathrm{~km}$, which is reached $\sim 75 \mathrm{~km}$ toward the center from the rim. The centermost $\sim 180 \mathrm{~km}$ of Tirawa is occupied by a set of ridges and plateaus which form a crudely circular pattern, much like the interior ridges of Odysseus, thus making Tirawa another putative "peak-ring basin" analog. Individual ridges are typically $\sim 50 \mathrm{~km}$ across at their base and 1-3 km high. Though Tirawa is roughly comparable in size with Odysseus, the impact that created it appears to have had comparatively less effect on Rhea beyond the outer rim of the impact feature. Tirawa's diameter is less than $25 \%$ of Rhea's diameter and the kinetic energy from the impact is $<0.1-1 \%$ of the satellite's gravitational binding energy, and only $\sim 15-20 \%$ of Tirawa's ejecta should have exceeded Rhea's escape velocity. Figure 9 includes high-resolution $\left(1 \mathrm{~km}_{\text {pixel }}{ }^{-1}\right)$ coverage of part of the circum-Tirawa region. There are a few small troughs $(\sim 5 \mathrm{~km}$ wide, $\sim 100 \mathrm{~km}$ long) and coalescing pits radial to the impact feature that might have been formed by Tirawa secondaries. In the same region there are more prominent coalescing pit chains, such as $\mathrm{Pu}$ Chou Chasma, that have no apparent relationship to Tirawa (Fig. 9) but may point to another impact feature (see below). Several small craters are apparent in the interior of Tirawa (Fig. 2c), suggesting that this may be a relatively old structure, and ejecta and secondaries from it are unlikely to be well preserved given the heavy cratering history of Rhea.

Tirawa's antipode (Fig. 10) was imaged by Voyager at moderate $\left(2 \mathrm{~km} \mathrm{pixel}^{-1}\right)$ resolution and low sun, revealing an undulatory, hilly surface. The anti-Tirawa region has possibly been resurfaced early in Rhean history (Smith et al., 1981; Moore et al., 1985) as evidenced by a difference in albedo with a distinct (though impact degraded) boundary separating this surface from a terrain dotted by larger craters (Verbiscer and Veverka, 1989; Buratti et al., 1990). All terrains on Rhea exhibit (to first order) saturation-equilibrium cratering for craters $\lesssim 30 \mathrm{~km}$ in diameter and the global occurrence of craters $\gtrsim 30 \mathrm{~km}$ in diameter is sufficiently sparse that their absence on the anti-Tirawa landscape could be due to impact-site randomness (Lissauer et al., 1988; cf. Section IV in McKinnon et al., 1991; Squyres et al., 1997). The only significant evidence at present for resurfacing of the anti-Tirawa region then is the albedo difference.
The undulating landscape of the anti-Tirawa region is consistent with, but not diagnostic of, a cratered terrain that has been somewhat degraded by seismic shaking. The degradation of this surface is not severe. Rims of craters in this area do not exhibit obvious scalloping or reduction to equidimensional mounds (at the resolution of the images) characteristic of severe seismic degradation on Mercury and the Moon (Schultz and Gault, 1975; Stuart-Alexander, 1978; Trask and Dzurisin, 1984), yet none are morphologically pristine. Indeed, the energy for seismically induced landform degradation at the level observed at the anti-Tirawa antipode may have been generated by local impacts and not necessarily be related to the Tirawa impact. Crossing this region are two light toned streaks that converge very near the antipode. Near the limit of resolution there are patterns consistent with coalescing pit chains running down the centers of the streaks, which might be formed by ejecta from the Tirawa impact (Fig. 10).

Long coalescing chains of oblong-to-teardropped-shaped pits cross much of the northern hemisphere well-imaged by Voyager 1 between $30^{\circ} \mathrm{W}$ and $120^{\circ} \mathrm{W}$, the most prominent of which include the named feature $\mathrm{Pu}$ Chou Chasma. These features were originally suggested by Moore et al. (1985) to be tectonic features. Here we interpret these features to be secondary crater chains, because they converge rather than run parallel and they exhibit raised rims. Our global mosaic of Rhea reveals that these pit chains and others less well imaged in the southern hemisphere all converge on a poorly imaged region that seems to be the site of a $\sim 350-\mathrm{km}-$ diameter annulus (centered at $\sim 25^{\circ} \mathrm{S}, 155^{\circ} \mathrm{W}$ ) (Fig. 11). The very strong resemblance of the coalescing pit chains to secondary crater chains, and the observation that they converge on a large circular annulus, lead us to infer that this annulus is a large impact feature of a size comparable with Tirawa. We infer that this putative large impact feature may be significantly younger than Tirawa, based on the relative abundance and morphologically pristine appearance of the coalescing pit chains we associate with the unnamed feature, compared with features we have associated with Tirawa ejecta.

The rolling surface of the region antipodal to the putative large impact feature is also consistent with, but not diagnostic of, a cratered terrain that has been degraded by some seismic shaking (Fig. 12), by the same criteria we employed in evaluating the anti-Tirawa region. As was the case with the anti-Tirawa region, the degradation of this surface is not severe and may prove to be characteristic of Rhea generally. The limitations of Voyager data preclude an unambiguous determination. Several pit-or-crater chains and a narrow trough (Kun Lun Chasma) converge very near the antipode. They are interpreted to be secondary crater chains because they exhibit raised rims. It is interesting to note that the two named chasmae ( $\mathrm{Pu}$ Chou and Kun Lun Chasmae) on Rhea lie on great circles radiating from the center of this putative large impact feature. 


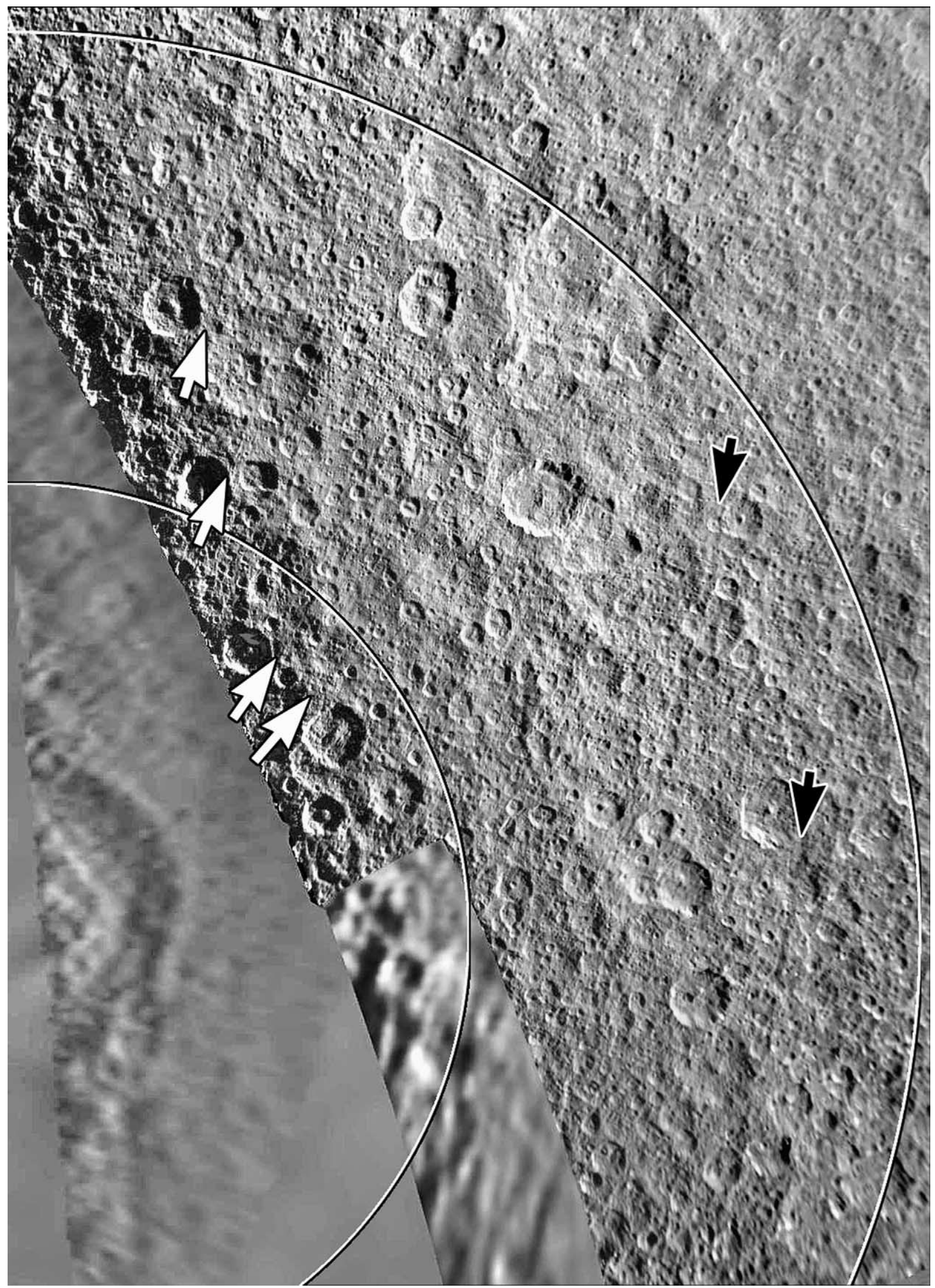

Fig. 9. Polar stereographic projection of a Voyager Rhea mosaic, centered on the Tirawa impact feature. The white arrows point to lineaments radial to Tirawa, and the black arrows point to Pu Chow Chasma (upper black arrow) and another lineament (lower black arrow) associated with a putative $\sim 350 \mathrm{~km}$-diameter impact feature at $25^{\circ} \mathrm{S}, 155^{\circ} \mathrm{W}$. The circles inscribe points $30^{\circ}$ and $60^{\circ}$ from Tirawa (for scale, $30^{\circ}$ equals $400 \mathrm{~km}$ in ground distance). 


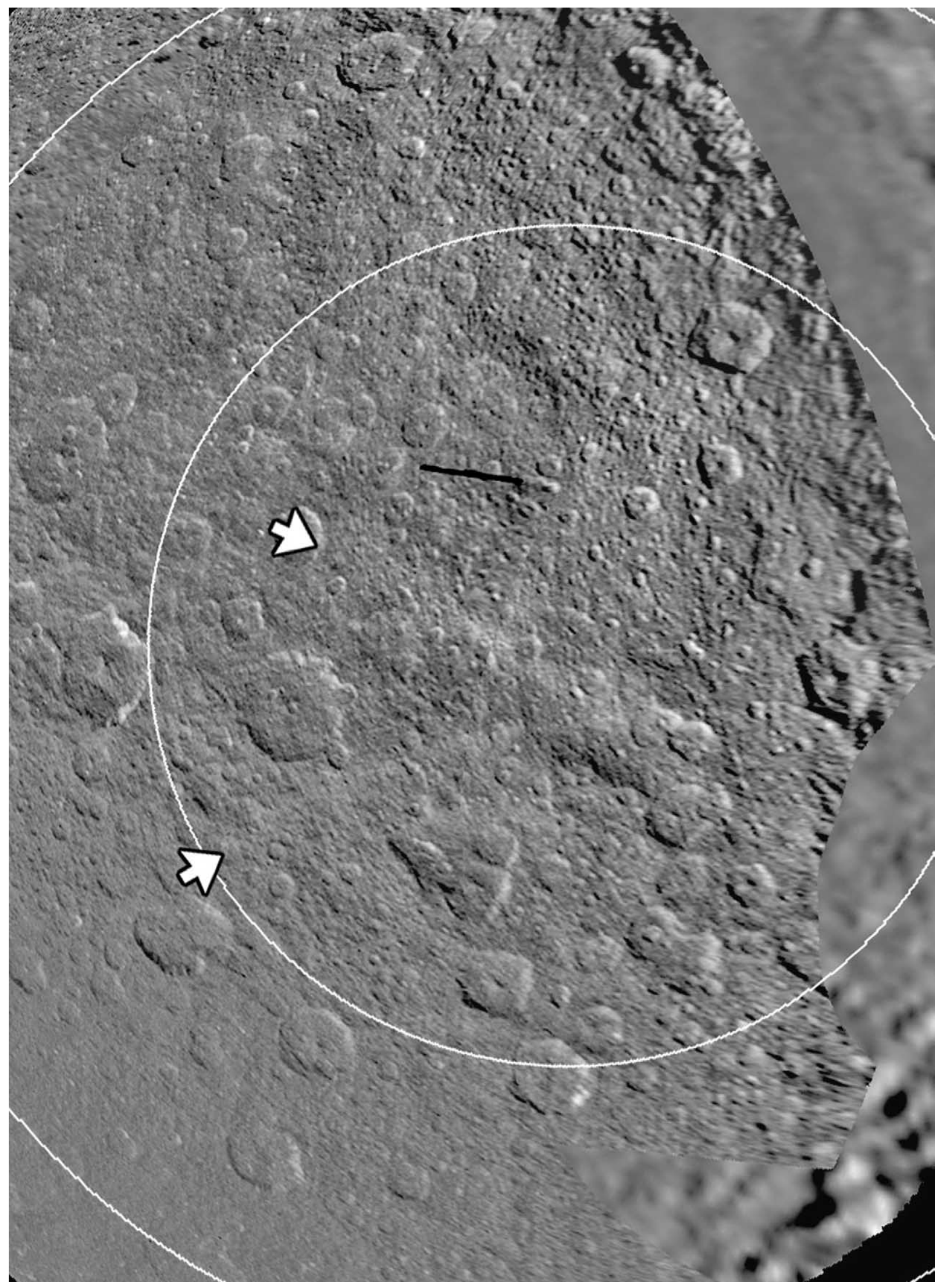

Fig. 10. Lambertian equal-area projection of a Voyager Rhea mosaic of the anti-Tirawa hemisphere centered on the Tirawa antipode $\left(36^{\circ} \mathrm{S}, 330^{\circ} \mathrm{W}\right)$. The circles inscribe points $30^{\circ}$ and $60^{\circ}$ from the Tirawa antipode (for scale, $30^{\circ}$ equals $400 \mathrm{~km}$ in ground distance). Arrows point at, and along the orientation of, light toned streaks more prominently seen in images taken at lower phase and incidence angle. 


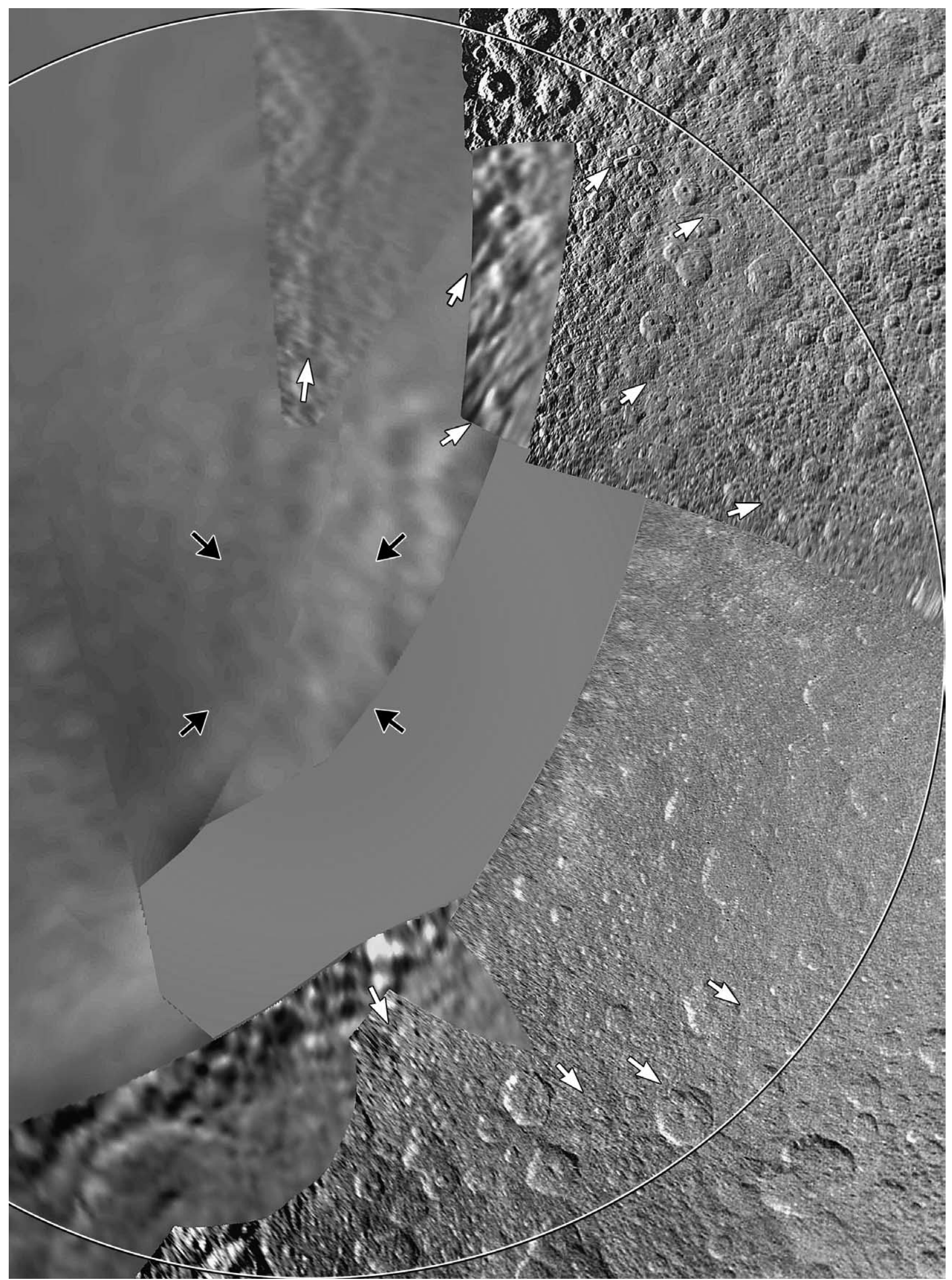

Fig. 11. Polar stereographic projection of a Voyager mosaic of Rhea, centered on a putative $\sim 350 \mathrm{~km}$-diameter impact feature at $25^{\circ} \mathrm{S}, 155^{\circ} \mathrm{W}$ (inscribed by the black arrows). The white arrows point to lineaments radial to this feature. The circle inscribes points $90^{\circ}$ from this feature (for scale, $90^{\circ}$ equals $1200 \mathrm{~km}$ in ground distance). 


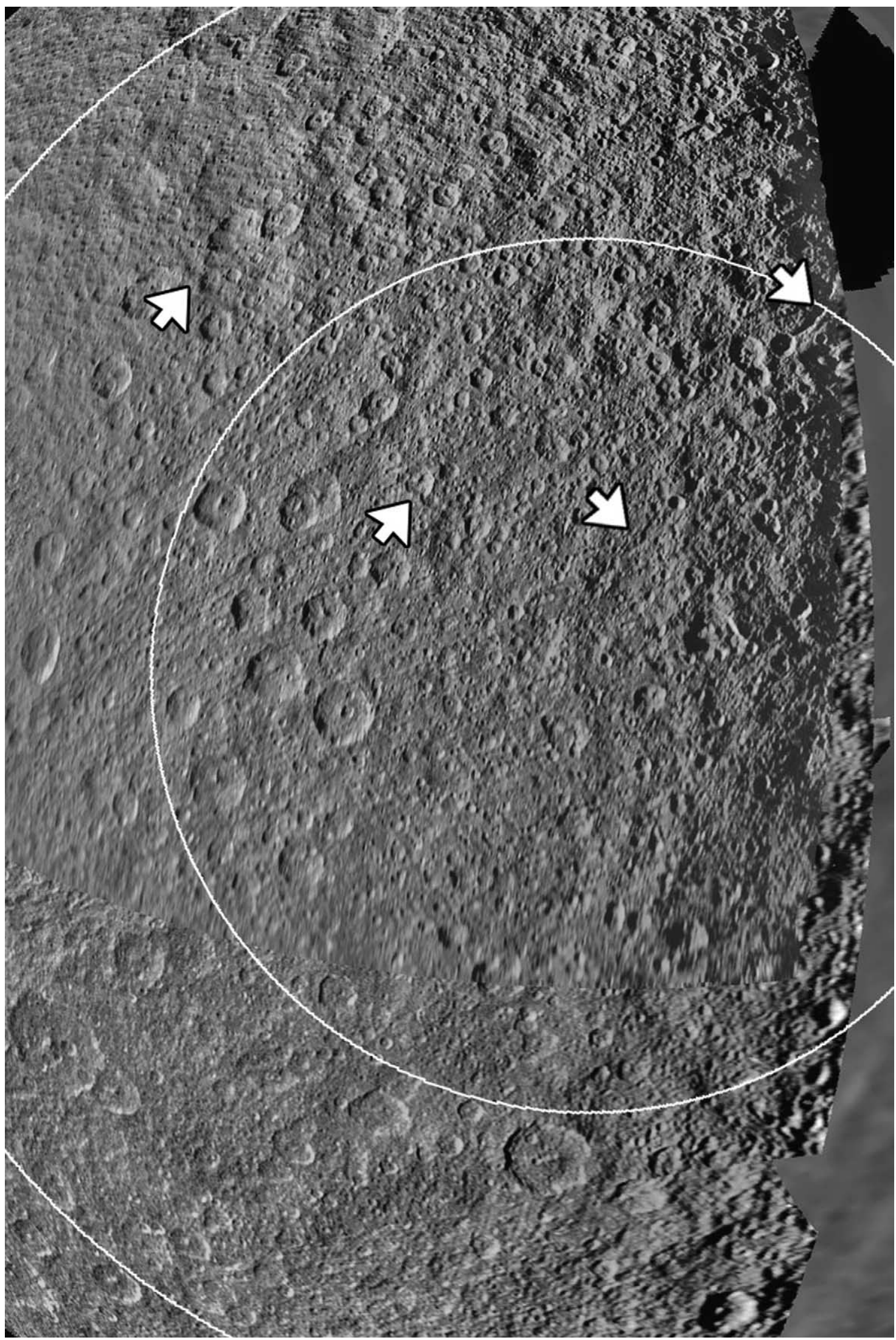

Fig. 12. Lambertian equal-area projection of a Voyager Rhea mosaic of the antipodal hemisphere of the putative $\sim 350 \mathrm{~km}$-diameter impact feature shown in Fig. 11, centered on that feature's antipode $\left(25^{\circ} \mathrm{N}, 335^{\circ} \mathrm{W}\right)$. Arrows point to linear pit chains that are oriented orthogonal to the direction of the arrows. The circles inscribe points $30^{\circ}$ and $60^{\circ}$ from the antipode (for scale, $30^{\circ}$ equals $400 \mathrm{~km}$ in ground distance). 


\subsection{Dione and Iapetus}

Saturnian satellites Dione (1118 km diameter) and Iapetus (1448 km diameter) both have large tracts of largecratered terrains. The largest craters on Dione putatively identified from Voyager images, elliptical Amata $(\sim 250 \mathrm{~km}$ diameter) and an unnamed feature identified by Plescia (1984) at $28^{\circ} \mathrm{N}, 257^{\circ} \mathrm{W}$ ( $200 \mathrm{~km}$ diameter), are located at the convergence of a hemisphere-wide network of high-albedo lineaments. The lineaments form bands that divide the surface into polygons (Fig. 1a). Moore (1984) interpreted this arrangement to be a manifestation of global extensional tectonism. Amata and its unnamed companion may have established deep-seated lines of weakness in Dione's lithosphere, which were later exploited by tectonic extension. We do note that the relatively smooth plains located at $\sim 60^{\circ}$ longitude and westward (Moore, 1984; Plescia, 1984), are roughly antipodal to Amata. No other impact structures have been identified in the Amata region, but this region was poorly observed by Voyager. Alternatively, "Amata" and the other putative structure nearby may prove to be illusory, quasi-circular features formed by the intersection of lineament sets.

A clear example of tectonic lineament orientations associated with an unambiguously identified impact feature is found around Turnus $\left(20^{\circ} \mathrm{N}, 345^{\circ} \mathrm{W}\right)$, which is only $90 \mathrm{~km}$ in diameter. Such crater-focused tectonism has been identified on Ganymede from Galileo data (e.g., Asphaug et al., 1998). If this crater-lineament relationship on Dione is real, then it appears to be unique among the middle-sized, icy satellites in this pre-Cassini survey.

The largest unequivocal impact on Dione, Aeneas, diameter $\sim 175 \mathrm{~km}$, is seen in our stereo data to be $3.5 \mathrm{~km}$ deep rim-to-floor (Figs. 2d and 3f), but possesses a large central peak $3.5 \mathrm{~km}$ high that extends $\sim 1.5 \mathrm{~km}$ above the mean local topography. In contrast, the next largest crater, Dido (diameter $\sim 140 \mathrm{~km}$ ) is $\sim 5 \mathrm{~km}$ deep. Dido and similar sized craters nearby are all relatively young compared to Aeneas, the floor of which has a number of small superposed craters. The relatively old and shallow floor of Aeneas is probably the most unequivocal case for viscous relaxation on any of the saturnian satellites. However, we do not see any evidence of circumferential or radial deformation associated with Aeneas.

Iapetus was not viewed at high resolution during the Voyager encounters and there are large gaps in available areal coverage, principally in the southern hemisphere. Moreover, little detail can be detected in the very dark (albedo $<0.05$ ) Cassini Regio. Thus, the only evidence for a large impact feature on Iapetus is a low-resolution $\left(23 \mathrm{~km} \mathrm{pixel}^{-1}\right)$ view of a dark annulus, centered on $5^{\circ} \mathrm{N}, 328^{\circ} \mathrm{W}$ with an outside diameter of $\sim 350 \mathrm{~km}$ and an inside diameter of half that value (Fig. 1a). In bright terrain regions, seen at moderate resolution $\left(8 \mathrm{~km} \mathrm{pixel}^{-1}\right)$, dark material can be seen filling depressions (Smith et al., 1982). If the $350 \mathrm{~km}$ dark annulus also resides in a depression, then it is reasonable to infer that it is the site of a large impact feature. The inner and outer diameter of the annulus has the same proportional spacing as between the putative peak-ring complexes and the rim crests of Odysseus and Tirawa, which may indicate that the Iapetus feature has a morphology similar to the other $400 \mathrm{~km}$-scale basins.

\subsection{Uranian satellites}

For completeness in our survey of middle-sized satellites, we review the evidence for large impact features on the uranian satellites. Nowhere in the Voyager imaging of the uranian satellites are seen large basins with the morphology of Odysseus or Tirawa. Of course, only one-third or so of these satellites' surfaces have been viewed, so such features may await discovery. What has been observed are several, probable large impact features that are apparently unlike those observed in the saturnian system.

Umbriel (1170 km diameter) displays a surface covered with large craters (Fig. 1b) apparently unaltered by endogenic processes (e.g., Smith et al., 1986). Thomas (1987) produced a limb profile of the highest resolution ( $5 \mathrm{~km} \mathrm{pixel}^{-1}$ ) image of Umbriel that shows a raise-rimmed depression (centered $45^{\circ} \mathrm{S}, 218^{\circ} \mathrm{E}$ ) $400 \mathrm{~km}$ from crest to crest, which is $\sim 6.5 \mathrm{~km}$ below the south rim and $\sim 5 \mathrm{~km}$ below the north rim. The center $175 \mathrm{~km}$ of the depression is occupied by a raised region bounded by peaks $\sim 1 \mathrm{~km}$ above the depression floor. Thomas (1988) and Croft and Soderblom (1991) interpreted this depression to be a large impact feature. An alternative explanation for this and other depressions seem in limb profiles of Umbriel are that they are large, low-lying crustal blocks, bounded by tectonically generated scarps (Helfenstein et al., 1989). The quality of the data prevents neither this nor the impact-basin explanation from being ruled out.

Titania (diameter $1578 \mathrm{~km}$ ) is the largest uranian satellite (Fig. 1b). The portion of its southern hemisphere imaged by Voyager is covered with craters, whose diameters rarely exceed 40 km (e.g., Smith et al., 1986). The Voyager imaging team interpreted the general absence of larger craters on Titania to indicate that the satellite had been extensively resurfaced, at least in the area seen by Voyager. Smith et al. (1986) tentatively identified large two impact features near the terminator in the highest resolution $\left(3 \mathrm{~km} \mathrm{pixel}^{-1}\right)$ images. The most probable is a $400 \pm 15 \mathrm{~km}$ diameter, raised-rim, circular feature named Gertrude $\left(17^{\circ} \mathrm{S}, 292^{\circ} \mathrm{E}\right)$. Stereo-derived topography of Gertrude (Figs. 2e and $3 \mathrm{~g}$ ) reveals that the rim is typical of an impact feature, with rim-to-floor relief of $\sim 2 \mathrm{~km}$ with an annular central "ring" or "dome" 2-3 km high, rising to elevations comparable to surrounding plains. The Gertrude "ring" or "dome" ( $150 \mathrm{~km}$ across) has only a few craters superimposed on it, prompting Smith et al. (1986) to map its surface as a site of a final episode of endogenic extrusion. The low relief of Gertrude and the absence of landforms related to impact ejecta raises the possibility that the surface on which 

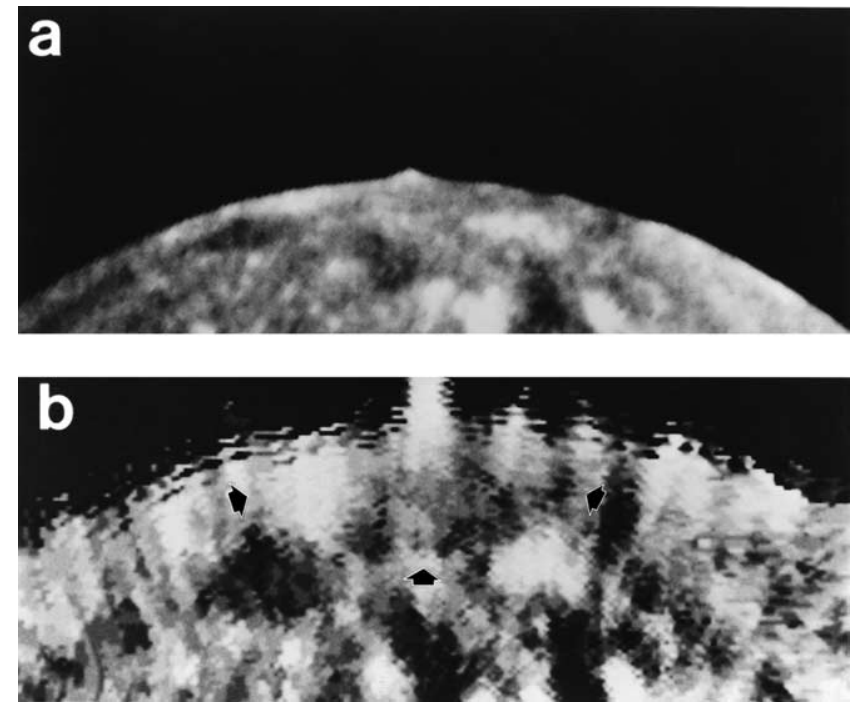

Fig. 13. The Oberon limb feature, centered at $23^{\circ} \mathrm{S}, 140^{\circ} \mathrm{E}$ : (a) original point-perspective view and (b) Polar projection of the same scene, centered on $23^{\circ} \mathrm{S}, 140^{\circ} \mathrm{E}$. The arrows in (b) point out the crude $\sim 375 \mathrm{~km}$ diameter semicircle discussed in the text. (Voyager image FDS 26836.23.)

the Gertrude rim was originally formed has been covered by subsequently emplaced materials or otherwise modified. Although some rim relief is preserved, Gertrude apparently has undergone significant viscous relaxation and/or volcanic modification.

Oberon (diameter $1522 \mathrm{~km}$ ) is covered by large ( $>40 \mathrm{~km}$ ) craters (e.g., Smith et al., 1986). Seen on its limb is a solitary peak (Figs. 1, 13), which Thomas (1987) has determined to be $11 \mathrm{~km}$ high and $45 \mathrm{~km}$ wide (Fig. $3 \mathrm{~h}$ ). It has been suggested that this feature (located at $23^{\circ} \mathrm{S}, 140^{\circ} \mathrm{E}$ ) is the central peak of a relaxed impact feature (Smith et al., 1986; Croft, 1987; McKinnon et al., 1991; Croft and Soderblom, 1991). The highest resolution $\left(6 \mathrm{~km} \mathrm{pixel}^{-1}\right)$ view of this feature transformed to a planometric projection (Fig. 13b) shows several dark patches whose boundaries collectively form a crude semicircle $\sim 375 \mathrm{~km}$ across. The limb profile reveals two 1-2 km high peaks, symmetric about the $11 \mathrm{~km}$ peak. These smaller peaks occur at the same distance from the large peak as does the albedo-defined semicircle. It is unlikely that some process other than impact cratering could have formed an isolated $11 \mathrm{~km}$ peak on Oberon. Taken together, the $11 \mathrm{~km}$ peak and the existence of a $\sim 375 \mathrm{~km}$ semicircular feature, composed of ridges where seen on the limb, indicate the presence of a large impact feature.

A $375-\mathrm{km}$ diameter central peak crater on Oberon may be anomalous with respect to crater morphologies of other icy satellites. If the transition from central peak to central pit craters is inversely correlated with surface gravity, as is the case for the simple-to-complex transition (e.g., Schenk, 1991; Schenk et al., 2004), then the largest central peak crater on Oberon should be only $\sim 200 \mathrm{~km}$ across. By this standard, the $375-\mathrm{km}$ crater on Oberon should more resemble Odysseus or Tirawa. By contrast, the central peak of Aeneas, $175 \mathrm{~km}$ across, is $4 \mathrm{~km}$ high. Simple scaling from
Aeneas suggests that this $11 \mathrm{~km}$ high peak could easily be a central peak of a very large impact crater if central peaks form in craters of these diameters. Without a clear image of this structure, however, little can be said about its true morphology, except that it is unlike any other crater to date seen in the outer Solar System.

For the sake of completeness, we briefly acknowledge speculation regarding large impact features on Miranda and Ariel, which otherwise have no unequivocally observed large impact features. It has been suggested (Croft, 1987; Croft and Soderblom, 1991; Greenberg et al., 1991) that Arden Corona, an approximately $320 \mathrm{~km}$-diameter ovoid centered at $15^{\circ} \mathrm{S}, 50^{\circ} \mathrm{E}$ on Miranda (diameter $472 \mathrm{~km}$ ) is the site of a very large impact. Though the interior of this feature appears to have been extensively modified by tectonic and possibly volcanic activity, the occurrence of landforms interpreted as secondary impact features, such as radially arranged coalescing pit chains and troughs that widen toward the ovoid, are cited by them as evidence for this hypothesis. It has also been proposed that a surficial mantle, through which only larger pre-existing craters can be detected, is the ejecta of the Arden site impact. Croft (1987) suggested that a $245 \mathrm{~km}$-diameter, roughly circular depression located at $10^{\circ} \mathrm{S}, 30^{\circ} \mathrm{E}$ on Ariel (diameter $1158 \mathrm{~km}$ ) is also the site of a large impact. The portion of the feature seen in the images is surrounded by sub-radial coalescing pit chains and troughs, which he interprets to be ejecta-created landforms. McKinnon et al. (1991) criticized this secondary crater hypothesis for Miranda (and by inference Ariel) as being inconsistent with impact physics as presently understood, in that impact velocities at these locations would be too slow to produce large ( 5-10 km-diameter) secondary craters. The interior of the feature is covered with texturally smooth, presumably extruded material.

\section{Discussion}

\subsection{Transient diameters of large impact craters}

Insight into the formation of large impact craters on middle-sized icy satellites, the amount of material they mobilize, and the depth of the disturbance into the satellite's interior can be appreciated by an estimation of the transient diameter of these craters during their excavation stage. There are no existing final/transient crater diameter scaling relations when the crater diameter is a large fraction of the target's diameter, so we use available expressions which assume an infinite half-space. McKinnon and Schenk (1995) presented an expression for transient crater diameter $\left(D_{\mathrm{tr}}\right)$ for impacts in ice as a function of the final rim diameter $\left(D_{\mathrm{f}}\right)$, based on the depth/diameter geometry of simple and complex craters and the simple-to-complex transition diameter $\left(D_{\mathrm{sc}}\right)$ for craters on Ganymede as determined by Schenk (1991): $D_{\mathrm{f}} \sim 1.176 D_{\mathrm{tr}}^{1.108}$ (with all diameters in $\mathrm{km}$ ). This is an expression specific to Ganymede, of a more general scal- 
Table 1

Derived simple-to-complex crater diameters $\left(D_{\mathrm{Sc}}\right)$ for several saturnian satellites

\begin{tabular}{lcll}
\hline Target & Surface $g\left(\mathrm{~cm} \mathrm{~s}^{-2}\right)$ & $D_{\text {sc }}$, scaled to $g$ & $D_{\mathrm{sc}}$, Schenk $(1991)$ \\
\hline Ganymede & 142.9 & $2 \mathrm{~km}$ & \\
Mimas & 7.9 & $36.2 \mathrm{~km}$ & $16(+17.7,-2.4)$ \\
Tethys & 18.5 & $15.4 \mathrm{~km}$ & 26 \\
Rhea & 28.5 & $9.97 \mathrm{~km}$ & $8.6(+12.7,-5.1)$ \\
\hline
\end{tabular}

See text for derivations.

ing relation of the form $D_{\mathrm{f}}=A D_{\mathrm{sc}}^{-0.108} \times D_{\mathrm{tr}}^{1.108}$ (where $A$ is a constant). We retain both these expressions while using revised, post-Galileo values for the simple-to-complex crater diameters on Ganymede and Callisto from (Schenk, 2002), 2 and $2.5 \mathrm{~km}$, respectively. Thus solving for $A=D_{\mathrm{sc}}^{0.108} \times$ $1.176 \mathrm{~km}$, we get 1.267 for a $D_{\mathrm{sc}}$ of $2 \mathrm{~km}$. If we use the expressions above for Callisto (which McKinnon and Schenk (1995) stated was justified within the errors), we get 1.298 for a $D_{\mathrm{sc}}$ of $2.5 \mathrm{~km}$. From this, we adopt $A=1.28$, and so we have the revised expression $D_{\mathrm{f}}=1.28 D_{\mathrm{sc}}^{-0.108} \times D_{\mathrm{tr}}^{1.108}$ for any icy satellite. ${ }^{4}$ We can also estimate values for simpleto-complex crater diameters on middle-sized icy satellites using $D_{\mathrm{sc}}=2\left(g_{\text {Ganymede }} / g\right) \mathrm{km}$, where $g$ is surface gravity of a given body and $2 \mathrm{~km}$ is the Ganymede transition diameter from Schenk (2002). The results for Mimas, Tethys, and Rhea are given in Table 1, where they are compared with values reported in Schenk (1991). (Note that the transition diameters reported by Schenk (1991) could be in error by $20 \%$ and are likely to be revised post-Cassini.) The "new" simple-to-complex crater diameter result for Rhea, which was best seen by Voyager, matches the Schenk (1991) Rhea result within rather broad errors. This gives us some confidence in using these "new" results for Mimas and Tethys.

We now use $D_{\text {tr }}=\left(\left(D_{\mathrm{sc}}^{0.108} / 1.28\right) \times D_{\mathrm{f}}\right)^{1 / 1.108}$ to determine the transient crater diameters of large impact craters on middle-sized icy satellites. For Hershel (diameter $=135 \mathrm{~km})$ on Mimas we get $D_{\text {tr }}=95 \mathrm{~km}$, Odysseus (diameter $=$ $425 \mathrm{~km}$ ) on Tethys we get $D_{\mathrm{tr}}=246 \mathrm{~km}$, and Tirawa (diameter $=350 \mathrm{~km}$ ) on Rhea we get $D_{\mathrm{tr}}=198 \mathrm{~km}$. These transient crater diameters were used to scale the impactor diameters in Bruesch and Asphaug (2004), assuming an impactor velocity $\left(20 \mathrm{~km} \mathrm{~s}^{-1}\right)$ and density $\left(917 \mathrm{~kg} \mathrm{~m}^{-3}\right)$.

\subsection{Ejecta and secondaries}

None of the large impact features we will discuss in this section were imaged by Voyager at sufficient resolution to unequivocally discern their proximal ejecta blanket facies. However, Schenk and Ridolfi (2002) identify probable ejecta deposits of smaller craters on Dione and Rhea with similar rim-scaled dimensions as ejecta deposits on Ganymede and Callisto. Secondaries were also observed at the $80-\mathrm{km}$ crater

\footnotetext{
4 This expression can be further improved by re-solving for the $D_{\mathrm{f}}-D_{\mathrm{tr}}$ relation using the new Ganymede crater geometry data (Schenk, 2002), or even more directly, using Cassini crater shape data once it is obtained.
}

Yu-ti on Rhea, which was imaged at $1 \mathrm{~km}$ pixel $^{-1}$ and optimum solar incidence angle for landform recognition. Thus we assume that expressions used to describe ejecta behavior on icy galilean satellites can be applied to middle-sized icy satellites.

It is reasonable to assume that $\sim 50 \%$ of the total volume of ejecta falls within $\sim 1$ crater radii of the rim, and the falloff in ejecta thickness is steep $(\sim 1 \text { (radial distance) })^{-2.8}$ ) (e.g., Housen et al., 1983), as these values are very roughly correct for all gravity regime craters. Further afield, ejecta, in the form of coherent blocks can form secondary craters. The unambiguous secondary craters of the 24-km-diameter impact feature Pwyll $\left(25^{\circ} \mathrm{S}, 271^{\circ} \mathrm{W}\right)$ on the galilean satellite Europa demonstrate that impacts into icy targets indeed do eject coherent blocks from coherent surfaces (Bierhaus et al., 2001) (although the low gravities of the middle-sized satellites means that many of these blocks will be lost). The strong resemblance of $\mathrm{Pu}$ Chou Chasma on Rhea, with its coalescing chains of oblong-to-teardrop-shaped pits, to secondary crater chains seen on many worlds indicates that impact ejecta from large impacts on the middle-sized icy satellites can also be coherent, and be derived from coherent surfaces. Ice at impact strain rates is a brittle solid, particularly at temperatures appropriate to the outer Solar System. Laboratory experiments (e.g., Lange and Ahrens, 1987) indicate a material that is dynamically very weak under tension. To get some sense of minimum velocities at which secondary craters form on icy targets, we examined high resolution, low sun Galileo images of the region around Europan crater Pwyll and identified the closest secondary craters. Pywll has a proximal pedestal ejecta facies that probably overrode the very closest secondaries (Moore et al., 1998), thus the closest one we measured was $40 \mathrm{~km}$ from Pwyll's center (Alpert and Melosh, 1999). Using the equation taken and rearranged from Melosh (1989, p. 87) for the velocity necessary to reach a short, specific ballistic range

$V=\sqrt{\frac{r g}{\sin 2 \theta}}$,

where $V$ is the secondary velocity at launch (and impact), $r$ is the range traveled by the secondary, $g$ is the surface gravity (which in Europa's case is $1.31 \mathrm{~m} \mathrm{~s}^{-2}$ ), and $\theta$ is the launch angle (taken to be $45^{\circ}$ ), we get $\sim 200 \mathrm{~m} \mathrm{~s}^{-1}$ for a range of $30 \mathrm{~km}$ and $\sim 230 \mathrm{~m} \mathrm{~s}^{-1}$ for a range of $40 \mathrm{~km}$.

To evaluate the validity of our secondary impact interpretation for candidate features on the middle-sized icy satellites, we first note the distance (or range) of the proximal ends of putative secondary crater chains from the centers of their presumed parents. For Odysseus on Tethys the distance is $\sim 220 \mathrm{~km}$. We use the equation for velocities of secondaries traveling over large ballistic ranges relative to the radius of the target taken from Haskins et al. (2003)

$$
V=\frac{\sqrt{R g \tan \left(\frac{r}{2 R}\right)}}{\sqrt{\tan \left(\frac{r}{2 R}\right) \cos ^{2} \theta+\sin \theta \cos \theta}},
$$


where the new term $R$ is the radius of the target planetary body. Using the just stated range value for putative secondary crater features on Tethys, we get a velocity of $165 \mathrm{~m} \mathrm{~s}^{-1}$. Ejecta moving at $200 \mathrm{~m} \mathrm{~s}^{-1}$ on Rhea will travel $\sim 170 \mathrm{~km}$ (or $12.6^{\circ}$ ), which is a shorter distance than Voyager resolutions permit for analysis of secondaries from either Tirawa or the putative $\sim 350 \mathrm{~km}$ basin. Thus all the putative secondary impact features we have identified on that satellite could be legitimate, and the most proximal secondary craters from these large impact features have probably not been seen. Blocks from Herschel on Mimas ejected at $100 \mathrm{~m} \mathrm{~s}^{-1}$ would travel $232 \mathrm{~km}$ ( or $68^{\circ}$ ) before striking the surface. As Mimas' escape velocity is $160 \mathrm{~m} \mathrm{~s}^{-1}$, it is possible that most material with velocities capable of forming secondary craters would initially escape this satellite and not form clusters or strings of secondaries that could be related to a given impact through geometry. These blocks would in large measure reimpact Mimas on short dynamical time scales, however (cf., Alvarellos et al., 2002). Much of the relatively slowly-moving, secondary ejecta falling back on direct trajectories on Mimas may simply form a deposit of rubble whose principle effect on pre-existing topography is a relief-muting mantle, much like the proximal ejecta blanket around craters on targets with higher gravity, but thinner and sparser.

\subsection{Global seismic effects}

We first consider the issue of disruption depth from extreme global seismic events, assuming these events were energetic enough to open fractures in the brittle ice (brittle on the time scale of these events) composing the interiors of the target satellites everywhere the lithostatic pressure was less than the brittle tensile strength of cold ice. Brittle fracture from global seismicity extends deeper into smaller satellites, all else being equal, as interior pressure shelters larger satellites from dynamic rupture. These being transient events, a simple estimate for the realm where brittle fracture may be expected to be induced, and visibly sustained, by global seismicity can be obtained by applying an expression (e.g., Turcott and Schubert, 2002, p. 85) for the lithostatic pressure at radius $R_{\mathrm{p}}$, and comparing this with the brittle tensile strength $(\sigma)$ of water ice at appropriate time scales and temperatures. To determine the depth of the fracturing we use laboratory-derived dynamic values of $\sim 1 \mathrm{MPa}$ (Lange and Ahrens, 1987; Hawkes and Mellor, 1972) at the appropriate temperatures, and using the following equation:

$R_{\mathrm{p}}=\sqrt{\frac{-\sigma}{\frac{2}{3} \pi G \rho^{2}}+R^{2}}$,

where $\rho$ is (bulk) density of the body (we approximated, using $1000 \mathrm{~kg} \mathrm{~m}^{-3}$ ), $G$ is the gravitational constant, and $R$ is the radius of the satellite. From this the value of $R-R_{\mathrm{p}}$ for Mimas is $\sim 20 \mathrm{~km}$, while that for Tethys is $\sim 10 \mathrm{~km}$. This is one measure of the depth to which it is reasonable to ex- pect the surface layer to intensity fractured (i.e., the megaregolith). Of course, a body may be fractured to greater depths by large impacts, and the resulting pore space may not easily be eliminated. Deep porosity is most easily maintained on smaller and presumably colder bodies such as Mimas (Eluszkiewicz, 1990). Thus for Mimas we expect relatively late-occurring major impact events, such as Herschel, might exploit pre-existing fracture patterns in the "megaregolith," which could persist deep into the body, rather than to refractively focus energy. Hence the lack of antipodal focusing or axial symmetry about a radius through Herschel in the lineament pattern on Mimas may be unsurprising, if that pattern is indeed an expression of tectonic movements initiated by the Herschel impact.

On the other hand, much of Tethys' radius could act as a mechanically coherent refractive focuser, as only the outer $2 \%$ has a lithostatic pressure less than the brittle tensile strength of ice. ${ }^{5}$ In order to investigate the effects of impact energy focusing on middle-sized icy satellites, Bruesch and Asphaug (2004) modeled antipodal focusing during giant impacts using a 3D Smooth Particle Hydrodynamics (SPH) impact code. That paper developed a method for modeling giant impact events in 3D targets and for studying resulting antipodal effects and global surface stresses. That paper also addressed the general problem of antipodal focusing as a function of satellite diameter and core/mantle composition, with case studies applied to several bodies including Tethys. Here we present a more comprehensive analysis of the Bruesch and Asphaug (2004) Tethys modeling results in the context of the observational constraints presented above.

$\mathrm{SPH}$ is a three-dimensional gridless Lagrangian algorithm (e.g., Benz, 1990; Monaghan, 1992) capable of incorporating realistic shape models (cf., Asphaug et al., 1998). The version of SPH used in this study has a unique fracture implementation (Benz and Asphaug, 1994, 1995), making it ideal for modeling geologic surface damage in solid body collisions. Models consisted of a target body and an impactor undergoing hypervelocity collision. To investigate the global impact effects in the simulations, the peak surface velocity and peak surface tensile stress were calculated for a particular location during an entire simulation. The degree to which the target lithosphere actually disrupts, however, is best answered by examining the magnitude of the peak stress and peak surface velocity during the course of the calculation.

Peak surface velocity indicates the magnitude of seismic shaking and the degree of terrain degradation. For degradation of terrains held together by gravity (deeply faulted mountains, ejecta deposits) the magnitude of degradation (expressed in terms of distance thrown, normalized to target radius) scales with $v_{\text {ej }} / v_{\text {esc }}$ where $v_{\text {ej }}$ is the peak surface velocity and $v_{\text {esc }}$ is the escape velocity at the surface. Peak surface tensile stress is a measure of material failure, faulting and spallation. Here peak surface tensile stress is only

\footnotetext{
5 If the smooth plains antipodal to Odysseus are indeed cryovolcanic, then Tethys was warm enough to have eliminated any deep porosity.
} 

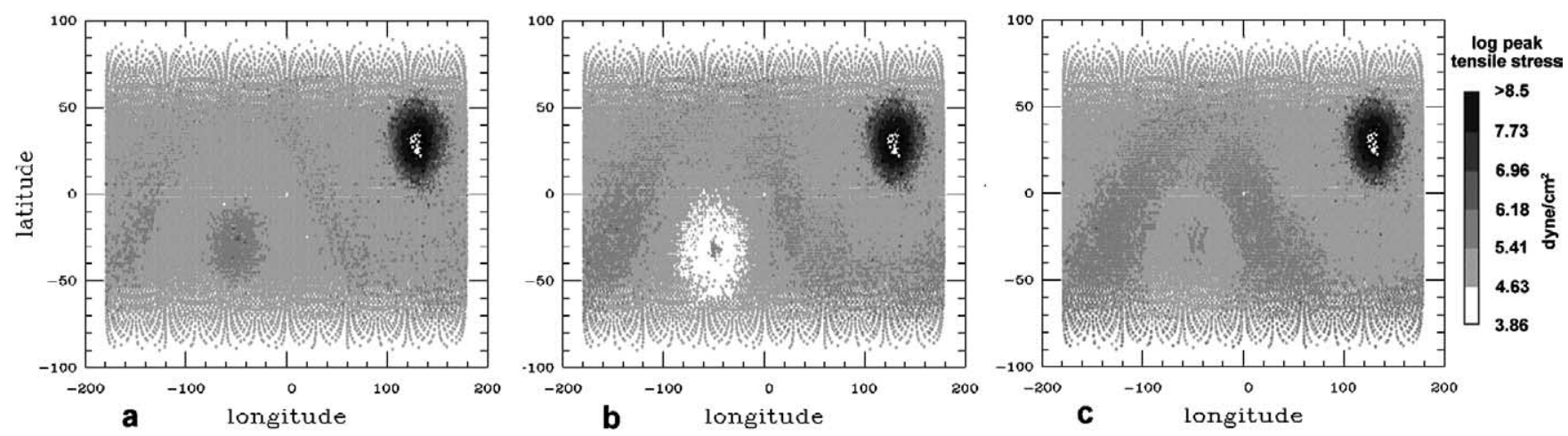

Fig. 14. Peak tensile stress in SPH models of Tethys following the Odysseus-forming impact event for three different core densities of (a) 2.0, (b) 2.7, and (c) $3.97 \mathrm{~g} \mathrm{~cm}^{-3}$, holding impact energy, impact angle, and bulk density $\left(1.26 \mathrm{~g} \mathrm{~cm}^{-3}\right.$ in these simulations) constant (see Bruesch and Asphaug (2004) for model details). Note that in all three cases there is enhanced (but modest) peak tensile stress around a great circle normal to the impact site, supportive of the hypothesis of Moore and Ahern (1983) that Ithaca Chasma was a prompt consequence of the Odysseus impact.

qualitatively applied at as an indicator of the propensity for surface rupture at any given location on the satellite's surface relative to another.

Compared to model results of Mimas, Rhea and a fiducial $1000 \mathrm{~km}$-diameter icy satellite, the model results for the Odysseus impact on Tethys show both more antipodal focusing and greater values of peak surface velocity and peak surface tensile stress in the antipodal hemisphere than any other simulation, as one might expect given its larger crater diameter relative to satellite diameter. Core density plays a role in global damage; the simulations incorporating a core density of $3970 \mathrm{~kg} \mathrm{~m}^{-3}$ caused significantly more widespread damage than did the simulations with core density of $2700 \mathrm{~kg} \mathrm{~m}^{-3}$ (see Fig. 14). The simulations were run for a bulk density of $1260 \mathrm{~kg} \mathrm{~m}^{-3}$ (Burns, 1986), whereas resonant dynamical estimates of Tethys' mass imply a density close to $1000 \mathrm{~kg} \mathrm{~m}^{-3}$ (Campbell and Anderson, 1989; Dourneau and Baratchart, 1999). However, the accuracy of the dynamical estimate has been questioned (i.e., it may be an underestimate; Thomas and Dermott, 1991). Other core and mantle configurations are easily implemented in the code, and detailed imaging and more accurate densities from Cassini will motivate an endeavor to use this model to reveal candidate interior structures as revealed by surface expressions of giant impacts.

As noted above, the antipode of Odysseus on Tethys consists of relatively smooth plains, but this plains unit may be the result of cryovolcanic resurfacing subsequent to the Odysseus impact. The Odysseus impact modeling also shows a hoop of enhanced peak tensile stress around the impact equator (Fig. 14). This supports the hypothesis of Moore and Ahern (1983) and Schenk (1989) that Ithaca Chasma is the result of the impact event that formed Odysseus because Ithaca conforms closely to the impact equator. However, instead of being the result of seismic disruption, Ithaca Chasma may be the result of whole body strain circumferential to the impact axis (Moore and Ahern, 1983), or a reflection of stress channeling from a relatively shallow core-mantle boundary. Further modeling work will be necessary to establish whether the maximum tensional principal stress is perpendicular to the impact equator, as it should be to cause the formation of such a rift, and whether the magnitude of the tensional stress is sufficient to cause faulting at depth (Cassini images will be important in establishing the depth of faulting).

The Odysseus impact modeling also shows that as the impact angle becomes more grazing, the hoop of enhanced peak tensile stress not only shifts slightly in longitude but also becomes more asymmetric. In the simulations with the largest impact angle $\left(0^{\circ}\right.$ is defined as normal to the impacted surface) one can clearly see higher values of peak tensile stress are present closer to the impact crater. The asymmetry in the values of peak tensile stress becomes apparent for impact angles greater than about $45^{\circ}$. This indicates that the asymmetry of the location of Ithaca Chasma and the smooth plains with respect to Odysseus antipode may be the result of an impact occurring at an angle greater than $45^{\circ}$. On the other hand, a highly oblique impact imparts less energy during an impact event, for fixed impactor characteristics, which results is less damage to the target body (i.e., lower values of peak surface velocity and peak surface tensile stress). Therefore, a very high impact angle, say greater than $80^{\circ}$, is not expected to have lead to the formation of Ithaca Chasma, subject to the uncertainty as to how the impactor size would scale to create Odysseus in such circumstances. An asymmetric core might also explain this phenomenon, however, this is not expected in bodies the size of Tethys. Unless Cassini returns a totally unexpected moment of inertia, the asymmetry seen in Ithaca Chasma with respect to a great circle normal to the center of Odysseus is likely due to an impact at an angle greater than $45^{\circ}$, if indeed the formation of Ithaca Chasma is a prompt effect. Evidence for oblique impact should be evident in the detailed morphology of Odysseus and its ejecta blanket that Cassini data may provide.

The Tirawa impact on Rhea was also modeled, the results of which indicated that this impact would focus significantly less energy at the antipode than was produced by the 
Odysseus impact on Tethys. This is not surprising in light of the smaller ratio of the final crater diameter to satellite diameter for Tirawa to Rhea compared with Odyssues to Tethys. Though not modeled, it is reasonable to speculate that the putative $\sim 350$-km-diameter impact feature on Rhea, being similar in size to Tirawa, would produce similar amounts of antipodal seismic shaking as did Tirawa. As we observed, the antipodal regions of these two Rhean impacts are consistent with, although not demonstrative of, moderate seismic shaking, but are certainly not enough to reduce the landscape to a level plain. Finally, the largest credible impact features on the uranian satellites have diameters proportional to the diameters of the host satellite that are comparable to or smaller than the relative size of Tirawa to Rhea, and so it is reasonable to think that these uranian satellite impacts had effects less than or similar to that of Tirawa at their antipodes.

\section{Hypotheses tests with Cassini}

The imminent arrival of Cassini in the Saturn system offers the opportunity to test and differentiate among the various explanations and speculations covered in this study. Many of the measurements reported here are unavoidably imprecise due to the limits in resolution and areal coverage of Voyager data, and could be vastly improved by Cassini observations. Here we set out important observations that we hope Cassini can implement:

1. Determinations of the simple-to-complex transition diameters for the saturnian middle-sized icy satellites can be done with image coverage of a statistically meaningful sample at resolutions better than $100 \mathrm{~m} \mathrm{pixel}^{-1}$ and in stereo where there is at least $30^{\circ}$ difference in viewing geometries but under similar lighting.

2. Imaging of as many known or suspected large (> $200 \mathrm{~km}$-diameter) impact features, as well as those discovered during the course of the mission, on the saturnian middle-sized icy satellites as possible at resolutions better than $1 \mathrm{~km} \mathrm{pixel}^{-1}$ and in stereo, in order to characterize their geomorphologies and relative ages. This campaign would permit the evaluation of the degree to which the largest of these features resemble the peak-ring basins of terrestrial planets (but not present on galilean satellites), and at what diameter the transition to peak-ring basins occurs. Such knowledge can guide modeling of the upper tens-ofkilometers of these satellites' interior (e.g., McKinnon and Melosh, 1980; Schenk, 2002) and the role of surface gravity in crater collapse and final floor presentation (e.g., Melosh, 1989).

3. Imaging of Tethys at sufficient resolution (on order of $1 \mathrm{~km}$ pixel $^{-1}$ or better and with high incidence illumination) to map out the distribution of Odysseus ejecta in order to constrain an impact angle for the Odysseus-forming event. These data can be used to run higher resolution simulations, involving rigorous tracking of ejecta from the Odysseus (as mentioned in Bruesch and Asphaug, 2004) to gauge the obliquity of the impact.

4. Mapping of Ithaca Chasma, the floor of Odysseus, and the trailing hemisphere plains unit on Tethys at sufficient resolution (on order of $500 \mathrm{~m} \mathrm{pixel}^{-1}$ or better and with high incidence illumination) to determine their relative ages principally using crater statistics. If Ithaca Chasma and the floor of Odysseus appear to the same age, then this will lend support to the Moore and Ahern (1983) hypothesis that Ithaca Chasma's formation was an immediate manifestation of the impact event. Structural geological analysis will determine the likely depth of the Chasma's faults, another important mechanical constraint on formation models. The age of the plains unit relative to Odysseus will settle whether the plains unit could have formed by severe seismic degradation associated with the Odysseus impact, or else formed later perhaps by cryovolcanic resurfacing.

5. Imaging of the regions of the antipodes of Tirawa and the putative $\sim 350$-km-diameter impact feature on Rhea at sufficient resolution (on order of $250 \mathrm{~m} \mathrm{pixel}^{-1}$ or better and with high incidence illumination) to fully characterize the extent and distribution of possible antipodal damage associated with these impacts. Maps of the distribution of ejecta of these large impacts can constrain the impact angles for these crater-forming events. This, coupled with the antipodal damage assessment, could be used as a crude measure of tomography through modeling (e.g., Bruesch and Asphaug, 2004).

6. Image a few sample regions around large fresh impact craters (perhaps as transects) at sufficient resolution (on order of $25 \mathrm{~m} \mathrm{pixel}^{-1}$ or better and with high incidence illumination) to identify the onset of secondary crater formation and to search for and characterize proximal ejecta deposit morphology and the effect of this slow-moving material on pre-existing landscapes. A couple of good candidates would be Telemachus $\left(56^{\circ} \mathrm{N}, 340^{\circ} \mathrm{W}\right)$ on Tethys, and Creusa $\left(50^{\circ} \mathrm{N}, 77^{\circ} \mathrm{W}\right)$ on Dione. Such knowledge will aid in quantifying the role of strength and fracture in icy surface materials.

7. Comprehensive mapping of the lineament system on Mimas to determine if there is any symmetry with it and Herschel as a means of assessing whether the two are related, and if so, what implications that pattern might have for Mimas' interior.

\section{Acknowledgments}

We especially thank Nadine Barlow and Beau Bierhaus for their careful reviews. Their comments and suggestions significantly improved the quality of this manuscript. This project began in 1986, at which time J.M.M. received a great deal of assistance from workers at the USGS in Flagstaff, whom he thanks. Also, due to the long gestation of this study, J.M.M. acknowledges support from NASA Planetary Geology and Geophysics Program (PGG) grants NSGW-1, 
NSGW-132, and NASA's Galileo Project. L.S.B. and E.A. were supported by NASA PGG grant NAG5-8914. P.M.S. and W.B.M. were supported by NASA PGG grants, and W.B.M. by a Uranus Data Analysis Program grant. We thank Peter Thomas for providing us with limb profiles of Tethys and Oberon. Thanks goes to Carrie Chavez for her help with manuscript preparation, Pam Engebretson for her help with figure preparation, and to Sue Selkirk and Dan Ball, who did the original figures over fifteen years ago.

\section{References}

Alpert, A.J., Melosh, H.J., 1999. Fragment sizes of high-speed ejecta from a large impact on Europa. In: Proc. Lunar Planet. Sci. Conf. 30th. Lunar and Planetary Institute, Houston. Abstract \#1881 [CD-ROM].

Alvarellos, J.L., Zahnle, K.J., Dobrovolskis, A.R., Hamill, P., 2002. Orbital evolution of impact ejecta from Ganymede. Icarus 160, 108-123.

Asphaug, E., Melosh, H.J., 1993. The Stickney impacts of PHOBOS: a dynamical model. Icarus 101, 144-164.

Asphaug, E., Ostro, S.J., Hudson, R.S., Scheeres, D.J., Benz, W., 1998. Disruption of kilometre-sized asteroids by energetic collisions. Nature 393, 437-440.

Benz, W., 1990. Smooth particle hydrodynamics: a review. In: Buchler, J.R. (Ed.), The Numerical Modeling of Nonlinear Stellar Pulsations. Kluwer Academic, The Netherlands, pp. 269-288.

Benz, W., Asphaug, E., 1994. Impact simulations with fracture: I. Method and tests. Icarus 107, 98-116.

Benz, W., Asphaug, E., 1995. Simulations of brittle solids using smooth particle hydrodynamics. Comput. Phys. Commun. 87, 253-265.

Bierhaus, E.B., Chapman, C.R., Merline, W.J., Brooks, S.M., Asphaug, E., 2001. Pwyll secondaries and other small craters on Europa. Icarus 153, 264-276.

Boyce, J.M., Dail, A.L., Soderblom, L.A., 1974. Ages of the lunar nearside light plains and maria. In: Proc. Lunar Planet. Sci. Conf. 5th, pp. 11-23.

Bruesch, L.S., Asphaug, E., 2004. Modeling global impact effects on middle-sized icy bodies: applications to Saturn's moons. Icarus. In press.

Buratti, B.J., Mosher, J.A., Johnson, T.V., 1990. Albedo and color maps of the saturnian satellites. Icarus 87, 339-357.

Burns, J.A., 1986. Some background about satellites. In: Burns, J.A., Matthews, M.S. (Eds.), Satellites. Univ. of Arizona Press, Tucson, pp. $1-38$.

Campbell, J.K., Anderson, J.D., 1989. Gravity field of the saturnian system from Pioneer and Voyager tracking data. Astron. J. 97, 1485-1495.

Chapman, C.R., McKinnon, W.B., 1986. Cratering of planetary satellites. In: Burns, J.A., Matthews, M.S. (Eds.), Satellites. Univ. of Arizona Press, Tucson, pp. 492-580.

Collins, G.S., Melosh, H.J., Morgan, J.V., Warner, M.R., 2002. Hydrocode simulations of Chicxulub Crater collapse and peak-ring formation. Icarus 157, 24-33.

Croft, S.K., 1987. Crater morphology and morphometry on the uranian satellites. In: NASA TM, vol. 89810, pp. 6-8.

Croft, S.K., Soderblom, L.A., 1991. Geology of the uranian satellites. In: Bergstralh, J.T., Miner, L.D., Matthews, M.S. (Eds.), Uranus. Univ. of Arizona Press, Tucson, pp. 561-628.

Dermott, S.F., Thomas, P.C., 1988. The shape and internal structure of Mimas. Icarus 73, 25-65.

Dourneau, G., Baratchart, S., 1999. Masses and densities of the four inner major Saturn's satellites. Astron. Astrophys. 350, 680-684.

Eluszkiewicz, J., 1990. Compaction and internal structure of Mimas. Icarus 84, 215-225.

Gilbert, G.K., 1893. The Moon's face: a study of the origin of its features. Bull. Phil. Soc. Washington 12, 241-292.
Greenberg, R., Croft, S.K., Janes, D.M., Kargel, J.S., Lebofsky, L.A., Lunine, J.I., Marcialis, R.L., Melosh, H.J., Ojakangas, G.W., Strom, R.G., 1991. Miranda. In: Bergstralh, J.T., Miner, L.D., Matthews, M.S. (Eds.), Uranus. Univ. of Arizona Press, Tucson, pp. 693-735.

Hale, W., Head, J.W., 1979. Central peaks in lunar craters: morphology and morphometry. In: Proc. Lunar Planet. Sci. Conf. 10th, pp. 2623-2633.

Hale, W.S., Grieve, R.A.F., 1982. Volumetric analysis of complex lunar craters: implications for basin rim formation. J. Geophys. Res. 87, 6576.

Haskins, L.A., Moss, B.E., McKinnon, W.B., 2003. On estimating contributions of basin ejecta to regolith deposits at lunar sites. Meteor. Planet. Sci. 38, 13-33.

Hawkes, I., Mellor, M., 1972. Deformation and fracture of ice under uniaxial stress. J. Glaciol. 11 (61), 103-131.

Helfenstein, P., Thomas, P.C., Veverka, J., 1989. Evidence from Voyager II photometry for early resurfacing of Umbriel. Nature 338, 324-326.

Housen, K.R., Schmidt, R.M., Holsapple, K.A., 1983. Crater ejecta scaling laws: fundamental forms based on dimensional analysis. J. Geophys. Res. 88, 2485-2499.

Lange, M.A., Ahrens, T.J., 1987. The dynamic tensile strength of ice and ice-silicate mixtures. J. Geophys. Res. 88, 1197-1208.

Lissauer, J.J., Squyres, S.W., Hartmann, W.K., 1988. Bombardment history of the Saturn system. J. Geophys. Res. 93, 13776-13804.

McKinnon, W.B., 1985. Geology of icy satellites. In: Klinger, J., Benest, D., Dollfus, A., Smoluchowski, R. (Eds.), Ices in the Solar System. Reidel, Dordrecht, pp. 820-856.

McKinnon, W.B., 1999. Midsize icy satellites. In: Beatty, J.K., Petersen, C.C., Chaikin, A. (Eds.), The New Solar System, fourth ed. Sky Publishing, Cambridge, pp. 297-310.

McKinnon, W.B., Benner, L.A.M., 1989. Origin of Ithaca Chasma, Tethys, II: the importance of the lithosphere. In: Proc. Lunar Planet. Sci. Conf. 20th, p. 679.

McKinnon, W.B., Melosh, H.J., 1980. Evolution of planetary lithospheres: evidence from multiringed structures on Ganymede and Callisto. Icarus 44, 454-471.

McKinnon, W.B., Schenk, P., 1995. Estimates of comet fragment masses from impact crater chains on Callisto and Ganymede. Geophys. Res. Lett. 22, 1829.

McKinnon, W.B., Chapman, C.R., Housen, K.R., 1991. Cratering of the uranian satellites. In: Bergstralh, J.T., Miner, L.D., Matthews, M.S. (Eds.), Uranus. Univ. of Arizona Press, Tucson, pp. 629-692.

Melosh, H.J., 1976. On the origin of fractures radial to lunar basins. In: Proc. Lunar Planet. Sci. Conf. 7th, pp. 2967-2982.

Melosh, H.J., 1980. Tectonic patterns on a tidally distorted planet. Icarus 43, 334-337.

Melosh, H.J., 1989. Impact Cratering: A Geologic Process. Oxford Univ. Press, New York.

Monaghan, J.J., 1992. Smoothed particle hydrodynamics. Annu. Rev. Astron. Astrophys. 30, 543-574.

Moore, J.M., 1984. The tectonic and volcanic history of Dione. Icarus 59, 205-220.

Moore, J.M., Ahern, J.L., 1983. The geology of Tethys. J. Geophys. Res. 88, A577-A584.

Moore, J.M., Malin, M.C., 1988. Dome craters on Ganymede. Geophys. Res. Lett. 15, 225-228.

Moore, J.M., Horner, V.M., Greeley, R., 1985. The geomorphology of Rhea: implications for geologic history and surface processes. J. Geophys. Res. 90, C785-C795.

Moore, J.M., 17 colleagues, 1998. Large impact features on Europa: results from the Galileo nominal mission. Icarus 135, 127-145.

Moore, J.M., 25 colleagues, 2001. Impact features on Europa: results of the Galileo Europa Mission (GEM). Icarus 151, 93-111.

Moore, J.M., 11 colleagues, 2004. Callisto. In: Bagenal, F. (Ed.), Jupiter. Cambridge Univ. Press, Cambridge, UK. In press.

Pike, R.J., 1980. Geometric interpretation of lunar craters. US Geol. Surv. Prof. Paper 1046-C.

Plescia, J.B., 1984. The geology of Dione. Icarus 56, 255-277. 
Plescia, J.B., Boyce, J.M., 1983. Crater numbers and geological histories of Iapetus, Enceladus, Tethys and Hyperion. Nature 301, 666-670.

Schenk, P.M., 1985. Mimas grooves and catastrophic breakups revisited. Bull. Am. Astron. Soc. 17, 738-739.

Schenk, P.M., 1989. Crater morphology and modification on the icy satellites of Uranus and Saturn: depth/diameter and central peak occurrence. J. Geophys. Res. 94, 3815-3832.

Schenk, P.M., 1991. Ganymede and Callisto-complex crater formation and planetary crusts. J. Geophys. Res. 96, 15635.

Schenk, P.M., 2002. Thickness constraints on the icy shells of the galilean satellites from a comparison of crater shapes. Nature 417, 419-421.

Schenk, P.M., Bulmer, M.H., 1998. Origin of mountains on Io by thrust faulting and large-scale mass movements. Science 279, 1514-1517.

Schenk, P.M., Ridolfi, F.J., 2002. Morphology and scaling of ejecta deposits on icy satellites. Geophys. Res. Lett. 29. 31(1)-31(4).

Schenk, P.M., Chapman, C.R., Zahnle, K., Moore, J.M., 2004. Ages and interiors: the cratering record of the galilean satellites. In: Bagenal, F. (Ed.), Jupiter. Cambridge Univ. Press, Cambridge, UK. In press.

Schultz, P.H., Gault, D.E., 1975. Seismic effects from major basin formations on the Moon and Mercury. Moon 12, 159-177.

Smith, B.A., 26 colleagues (The Voyager Imaging Team), 1981. Encounter with Saturn: Voyager 1 imaging science results. Science 212, 163-191.

Smith, B.A., 28 colleagues (The Voyager Imaging Team), 1982. A new look at the Saturn system: the Voyager 2 images. Science 215, 504-537.

Smith, B.A., 39 colleagues (The Voyager Imaging Team), 1986. Voyager 2 in the uranian system: imaging science results. Science 233, 43-64.

Soter, S., Harris, A., 1977. The equilibrium figures of Phobos and other small bodies. Icarus 30, 192-199.

Spudis, P.D., 1982. The geology of lunar multi-ring basins. PhD dissertation. Arizona State University, Tempe.
Stuart-Alexander, D.E., 1978. Geologic map of the central far side of the Moon. US Geol. Surv. Misc. Inv. Map I-1047.

Squyres, S.W., Howell, C., Liu, M.C., Lissauer, J.J., 1997. Investigation of crater "saturation" using spatial statistics. Icarus 125, 67-82.

Thomas, P.C., 1987. The morphology of Phobos and Deimos. PhD dissertation. Cornell University, Ithaca.

Thomas, P.C., 1988. Radii, shapes, and topography of the satellites of Uranus from limb coordinates. Icarus 73, 427-441.

Thomas, P.C., Dermott, S.F., 1991. The shape of Tethys. Icarus 94, 391398.

Thomas, P.J., Squyres, S.W., 1988. Relaxation of impact basins on icy satellites. J. Geophys. Res. 93, 14919-14932.

Trask, N.J., Dzurisin, D., 1984. Geologic map of the discovery quadrangle of Mercury. US Geol. Surv. Misc. Inv. Map I-1658.

Turcott, D.L., Schubert, G., 2002. Geodynamics, second ed. Cambridge Univ. Press, Cambridge.

Verbiscer, A.J., Veverka, J., 1989. Albedo dichotomy of Rhea: Hapke analysis of Voyager photometry. Icarus 82, 336-353.

Veverka, J., Thomas, P.C., Johnson, T.V., Matson, D., Housen, K.R., 1986. The physical characteristics of satellites surfaces. In: Burns, J.A., Matthews, M.S. (Eds.), Satellites. Univ. of Arizona Press, Tucson, pp. 242-402.

Watts, A.W., Greeley, R., Melosh, H.J., 1991. The formation of terrains antipodal of major impacts. Icarus 93, 159-168.

Wilhelms, D.E., 1987. The geologic history of the Moon. US Geol. Surv. Prof. Paper 1348, 302.

Yoder, C.F., 1995. Astrometric and geodetic properties of Earth and the Solar System. In: Global Earth Physics: A Handbook of Geophysical Constants AGU Ref. Shelf, vol. 1, pp. 1-31. 